\title{
SOME REDUCTIONS OF RANK 2 AND GENERA 2 AND 3 HITCHIN SYSTEMS
}

\author{
OLEG K. SHEINMAN
}

\begin{abstract}
Certain reductions of the rank 2, genera 2 and 3 Hitchin systems are considered, which are shown to give an integrable system of 2 , resp. 3 , interacting points on the line. It is shown that the reduced systems are particular cases of a certain universal integrable system related to the Lagrange interpolation polynomial. Admissibility of the reduction is proved using computer technique. The corresponding codes are given in the text.
\end{abstract}

\section{Contents}

1. Introduction

2. Reduction, genera 2 and 3

3. Integrable system related to the Lagrange interpolation polynomial

4. Genus 2. Codes

5. Genus 3. Codes

References

\section{INTRODUCTION}

General procedures of finding the algebraic-geometric solutions, and the actionangle coordinates for Hitchin systems are proposed in [3]. For the rank 2 genus 2 systems the problem had been considered earlier in [1] based on the analogy with the Neumann system, and the results of [2]. However, if we ask what a dynamical system corresponds to a given Hitchin Hamiltonian 1 , say, in the Tyurin parametrization, we will find out that it is a problem even write down the corresponding equations because the expressions for the Hamiltonians consist of thousands of symbols.

In the present work we consider reductions of Hitchin systems, i.e. their restrictions to invariant subvarieties of a positive codimension. On this way we obtain some manageable rank 2, genera 2 and 3 systems, certain relations for their dynamical variables, and some particular solutions to the original systems.

It turns out to be that on the genus $g$ curve the reduced system is integrable and coincides with the universal integrable system related to the Lagrange interpolation polynomial of degree $g-1$.

The paper is organized as follows. In Section 2 we describe the reduction of the Hitchin systems of rank 2, genera 2 and 3 , prove it to be an admissible reduction, observe that the reduced systems are completely integrable and find some their particular solutions (which are solutions to the original Hitchin system as well). The

${ }^{1}$ The author is grateful to I.M.Krichever for this question 
proof of admissibility is computational. The codes of the corresponding programs are given in Sections 4 , 5

Section 3 is devoted to the integrable system related to the Lagrange interpolation polynomial.

The author is grateful to S.P.Novikov who multiply put the question of detailed investigation of Hitchin systems of small ranks and genera, and to I.M.Krichever for discussions of setting the problem and some results.

\section{Reduction, genera 2 And 3}

Let $\Sigma$ be a genus $g$ hyperelliptic curve given by the equation $y^{2}=P_{2 g+1}(x)$ where $P_{2 g+1}$ is a degree $2 g+1$ polynomial. Consider a Lax operator with the spectral parameter on this curve, taking values in the full linear algebra $\mathfrak{g l}(2)$, given in the Tyurin parametrization [3] (see [6]-11] for the further developments):

$$
L(x, y)=\sum_{i=0}^{g-1} L_{i} x^{i}+\sum_{s=1}^{2 g} \alpha_{s} \beta_{s}^{T} \frac{y+b_{s}}{x-a_{s}},
$$

where $\left(a_{s}, b_{s}\right) \in \Sigma, \alpha_{s}, \beta_{s} \in \mathbb{C}^{2}, \beta_{s}^{T} \alpha_{s}=0, L\left(a_{s}, b_{s}\right) \alpha_{s}=\kappa_{s} \alpha_{s}, \kappa_{s} \in \mathbb{C}$ for any $s=1, \ldots, 2 g$ (the upper $T$ denotes transposing here). Observe that there arise no singularity in the above eigenvalue conditions due to the assumption $\beta_{s}^{T} \alpha_{s}=0$, and also because the points $a_{s}(s=1, \ldots, 2 g)$ are assumed to be different. By fixing a gauge, we set the matrix formed by the vectors $\alpha_{2 g-1}, \alpha_{2 g}$ to the unit $2 \times 2$ matrix. Below, $g=2,3$.

A rank 2 Hitchin system on $\Sigma$ is given by the canonical symplectic structure $\omega=\sum_{s=1}^{2 g} \alpha_{s}^{T} \wedge \beta_{s}+\sum_{s=1}^{2 g} a_{s} \wedge \kappa_{s}$, and the Hamiltonians of the form

$$
H_{k, m}=\operatorname{res}_{z=0} z^{m-1} \operatorname{tr} L(z)^{k} \mathrm{~d} x(z) / y(z),
$$

where $z=\frac{1}{\sqrt{x}}$ is a local parameter in the neighborhood of the point $x=\infty$, and $k, m \in \mathbb{Z}$.

In the case $g=2$ we consider the canonical equations corresponding to the Hamiltonian $H_{2,2}$. Assuming $p_{1}=0$ where $y^{2}=x^{5}+p_{1} x^{4}+\ldots+p_{5}$ is the equation of the curve we state

Proposition 2.1. For a genus 2 curve $\Sigma$ the reduction $\alpha_{11}=\alpha_{22}=0, \beta_{1}=\beta_{2}=$ $\beta_{3}=\beta_{4}=0$ is admissible (compatible with the system), and the corresponding reduced system has the form

$$
\begin{array}{cc}
\dot{a}_{1}=-\frac{2\left(a_{1} \kappa_{4}+a_{4}\left(-2 \kappa_{1}+\kappa_{4}\right)\right)}{\left(a_{1}-a_{4}\right)^{2}}, & \dot{\kappa}_{1}=\frac{\kappa_{1}-\kappa_{4}}{a_{1}-a_{4}} \dot{a}_{1} \\
\dot{a}_{4}=-\frac{2\left(a_{4} \kappa_{1}+a_{1}\left(\kappa_{1}-2 \kappa_{4}\right)\right)}{\left(a_{1}-a_{4}\right)^{2}}, & \dot{\kappa}_{4}=\frac{\kappa_{1}-\kappa_{4}}{a_{1}-a_{4}} \dot{a}_{4} \\
\dot{a}_{2}=-\frac{2\left(a_{2} \kappa_{3}+a_{3}\left(-2 \kappa_{2}+\kappa_{3}\right)\right)}{\left(a_{2}-a_{3}\right)^{2}}, & \dot{\kappa}_{2}=\frac{\kappa_{2}-\kappa_{3}}{a_{2}-a_{3}} \dot{a}_{2} \\
\dot{a}_{3}=-\frac{2\left(a_{3} \kappa_{2}+a_{2}\left(\kappa_{2}-2 \kappa_{3}\right)\right)}{\left(a_{2}-a_{3}\right)^{2}}, & \dot{\kappa}_{3}=\frac{\kappa_{2}-\kappa_{3}}{a_{2}-a_{3}} \dot{a}_{3} \\
\dot{\alpha}_{12}=\alpha_{12} \frac{b_{2}+b_{3}}{a_{2}-a_{3}} \dot{a}_{3}, \quad \dot{\alpha}_{21}=\alpha_{21} \frac{b_{1}+b_{4}}{a_{1}-a_{4}} \dot{a}_{4} .
\end{array}
$$


The system of the first 8 equations (for the system of points) is Hamiltonian with the Hamiltonian

$$
\begin{aligned}
H_{2,2}^{(r)} & =2\left(\kappa_{1}-\kappa_{4}\right)\left(a_{4} \kappa_{1}-a_{1} \kappa_{4}\right) /\left(a_{1}-a_{4}\right)^{2} \\
& +2\left(\kappa_{2}-\kappa_{3}\right)\left(a_{3} \kappa_{2}-a_{2} \kappa_{3}\right) /\left(a_{2}-a_{3}\right)^{2}
\end{aligned}
$$

The proof consists of a direct calculation using the program "Wolfram Mathematica": it turns out to be that the derivatives of the corresponding variables by virtue of the system vanish after plugging $\alpha_{11}=\alpha_{22}=0, \beta_{1}=\beta_{2}=\beta_{3}=\beta_{4}=0$. The programs for this and other calculations are given below (in Section 4 for genus 2, and in Section 5 for genus 3).

The obtained system of equations splits into the two consistent subsystems: one of them for unknowns $a_{1}, a_{4}, \kappa_{1}, \kappa_{4}, \alpha_{21}$, and another for unknowns $a_{2}, a_{3}, \kappa_{2}$, $\kappa_{3}, \alpha_{12}$. Both can be resolved similarly. For example, we have for the first one $\frac{\dot{\kappa}_{1}-\dot{\kappa}_{4}}{\kappa_{1}-\kappa_{4}}=\frac{\dot{a}_{1}-\dot{a}_{4}}{a_{1}-a_{4}}$, which implies $\kappa_{1}=c_{1} a_{1}+c_{2}, \kappa_{4}=c_{1} a_{4}+c_{3}\left(c_{1}, c_{2}, c_{3}\right.$ are constants). For $c_{1}=c_{2}=c_{3}$ the arising equations for $a_{1}, a_{4}$ can be completely integrated: $a_{1}+a_{4}+2 a_{4}^{2}=c t+\tilde{\tilde{c}}, 2 a_{4}+\ln \left(a_{1}+a_{4}\right)+\tilde{c}=0$, where $c, \tilde{c}, \tilde{\tilde{c}}$ are constants. Next, the equation for $\alpha_{21}$ can be transformed to the form $d \ln \alpha_{21}=\frac{b_{1}+b_{4}}{a_{1}-a_{4}} d a_{4}$. The right hand side can be considered as a function of only $a_{4}$, which is, moreover, already known, and we have $\alpha_{21}=\exp \int \frac{b_{1}+b_{4}}{a_{1}-a_{4}} d a_{4}$.

Besides the reduction $\alpha_{11}=\alpha_{22}=0$ there is another one $\alpha_{12}=\alpha_{21}=0$ which descends to the previous by means of permutation of the points $a_{1}$ and $a_{2}$.

For a genus 3 curve

$$
y^{2}=x^{7}+p_{1} x^{6}+p_{2} x^{5}+p_{3} x^{4}+p_{4} x^{3}+p_{5} x^{2}+p_{6} x+p_{7}
$$

we fix a gauge by requiring that the vectors $\alpha_{5}$ and $\alpha_{6}$ form the unit matrix. Then for $p_{1}=p_{2}=p_{3}=0$ the reduced system splits to the two (independent) triples of interacting points, and the equations for the parameters $\alpha_{i j}$. For example for the Hamiltonian $H_{2,4}$ under the reduction

$$
\begin{gathered}
\alpha_{11}=0, \alpha_{22}=0, \alpha_{13}=0, \alpha_{24}=0, \\
\beta_{s}=0 \quad(s=1, \ldots, 6)
\end{gathered}
$$

we obtain for the points $a_{1}, a_{3}, a_{6}$ (for which the first coordinates of the corresponding vectors $\alpha_{s}$ are equal to zero) a Hamiltonian system with the Hamiltonian

$$
\begin{aligned}
& H_{2,4}^{(r)}=\frac{\left(a_{6}^{2}\left(-\kappa_{1}+\kappa_{3}\right)+a_{3}^{2}\left(\kappa_{1}-\kappa_{6}\right)+a_{1}^{2}\left(-\kappa_{3}+\kappa_{6}\right)\right)^{2}}{\left(a_{1}-a_{3}\right)^{2}\left(a_{1}-a_{6}\right)^{2}\left(a_{3}-a_{6}\right)^{2}}+ \\
& +\frac{2\left(a_{6}\left(\kappa_{3}-\kappa_{1}\right)+a_{3}\left(\kappa_{1}-\kappa_{6}\right)+a_{1}\left(\kappa_{6}-\kappa_{3}\right)\right)}{\left(a_{1}-a_{3}\right)\left(a_{1}-a_{6}\right)\left(a_{3}-a_{6}\right)} \times \\
& \times \frac{a_{6}^{2}\left(a_{1} \kappa_{3}-a_{3} \kappa_{1}\right)+a_{3}^{2}\left(a_{6} \kappa_{1}-a_{1} \kappa_{6}\right)+a_{1}^{2}\left(a_{3} \kappa_{6}-a_{6} \kappa_{3}\right)}{\left(a_{1}-a_{3}\right)\left(a_{1}-a_{6}\right)\left(a_{3}-a_{6}\right)}
\end{aligned}
$$

The following relations between $\dot{\kappa}_{i}$ and $\dot{a}_{i}$ hold:

$$
\begin{aligned}
& \dot{\kappa}_{1}=\frac{\left(a_{1}-a_{6}\right)^{2}\left(\kappa_{1}-\kappa_{3}\right)-\left(a_{1}-a_{3}\right)^{2}\left(\kappa_{1}-\kappa_{6}\right)}{\left(a_{1}-a_{3}\right)\left(a_{1}-a_{6}\right)\left(a_{3}-a_{6}\right)} \dot{a}_{1} \\
& \dot{\kappa}_{3}=\frac{\left(a_{3}-a_{6}\right)^{2}\left(\kappa_{1}-\kappa_{3}\right)+\left(a_{1}-a_{3}\right)^{2}\left(\kappa_{3}-\kappa_{6}\right)}{\left(a_{1}-a_{3}\right)\left(a_{1}-a_{6}\right)\left(a_{3}-a_{6}\right)} \dot{a}_{3} \\
& \dot{\kappa}_{6}=\frac{\left(a_{6}-a_{1}\right)^{2}\left(\kappa_{3}-\kappa_{6}\right)-\left(a_{6}-a_{3}\right)^{2}\left(\kappa_{1}-\kappa_{6}\right)}{\left(a_{1}-a_{3}\right)\left(a_{1}-a_{6}\right)\left(a_{3}-a_{6}\right)} \dot{a}_{6}
\end{aligned}
$$


Also we have:

$$
\dot{a}_{1}+\dot{a}_{3}+\dot{a}_{6}=\frac{2\left(a_{6}\left(-\kappa_{1}+\kappa_{3}\right)+a_{3}\left(\kappa_{1}-\kappa_{6}\right)+a_{1}\left(-\kappa_{3}+\kappa_{6}\right)\right)}{\left(a_{1}-a_{3}\right)\left(a_{1}-a_{6}\right)\left(a_{3}-a_{6}\right)} .
$$

The relations (2.2) are proven below (see Lemma 3.3 and after that). As for (2.3), it still is only a result of computation.

Under assumption $\kappa_{1}=\kappa_{3}=\kappa_{6}=K(=$ const $)$ we obtain the following equations:

and

$$
\begin{aligned}
& \dot{a}_{1}=\frac{2 K}{\left(a_{1}-a_{3}\right)\left(a_{1}-a_{6}\right)} \\
& \dot{a}_{3}=-\frac{2 K}{\left(a_{1}-a_{3}\right)\left(a_{3}-a_{6}\right)} \\
& \dot{a}_{6}=\frac{2 K}{\left(a_{1}-a_{6}\right)\left(a_{3}-a_{6}\right)}
\end{aligned}
$$

$$
\begin{aligned}
& \frac{\dot{\alpha}_{21}}{\alpha_{21}}=\frac{2 K\left(b_{1}+b_{6}\right)}{\left(a_{1}-a_{6}\right)^{2}\left(a_{3}-a_{6}\right)}-\frac{2 K\left(b_{1}+b_{3}\right)}{\left(a_{1}-a_{3}\right)^{2}\left(a_{3}-a_{6}\right)} \\
& \frac{\dot{\alpha}_{23}}{\alpha_{23}}=\frac{2 K\left(b_{3}+b_{6}\right)}{\left(a_{1}-a_{6}\right)\left(a_{3}-a_{6}\right)^{2}}-\frac{2 K\left(b_{1}+b_{3}\right)}{\left(a_{1}-a_{3}\right)^{2}\left(a_{1}-a_{6}\right)},
\end{aligned}
$$

in particular it follows that $\dot{a}_{1}+\dot{a}_{3}+\dot{a}_{6}=0$. The variables in the system of equations on $a_{s}$ do separate: setting

$$
a_{1}+a_{3}+a_{6}=C_{1}
$$

we obtain

$$
\left(\dot{a}_{1}-\dot{a}_{3}\right)\left(a_{1}-a_{3}\right)=-3\left(\dot{a}_{1}+\dot{a}_{3}\right)\left(a_{1}+a_{3}-2 C_{1} / 3\right)
$$

which is resolved in the form:

$$
\begin{aligned}
& a_{1}^{3}+f_{1} C_{1} a_{1}^{2}+f_{2} C_{1}^{2} a_{1}=-2 K t+C_{2}, \\
& a_{3}=e^{ \pm 2 \pi i / 3} a_{1}+f_{0} C_{1}, \quad a_{6}=C_{1}-a_{1}-a_{3},
\end{aligned}
$$

where $f_{0}, f_{1}, f_{2} \in \mathbb{C}$ are known coefficients (the explicit expressions for them are omitted here), $C_{1}, C_{2}$ are the integration constants. In particular, for $C_{1}=0$ we obtain

$$
a_{1}=\sqrt[3]{-2 K t+C_{2}}, \quad a_{3}=e^{ \pm 2 \pi i / 3} a_{1}, \quad a_{1}+a_{3}+a_{6}=0 .
$$

The variables $\alpha_{i s}$ can be expressed via quadratures, similar to the genus 2 case.

Proposition 2.2. The reduced systems are completely integrable. The corresponding full system of integrals in involution for $g=2$ (and the system of the points $\left.\left\{a_{1}, a_{4}\right\}\right)$ is as follows:

$$
F_{0}=\frac{a_{1} \kappa_{4}-a_{4} \kappa_{1}}{a_{1}-a_{4}}, \quad F_{1}=\frac{\kappa_{1}-\kappa_{4}}{a_{1}-a_{4}},
$$

and for $g=3$ as follows:

$$
\begin{aligned}
& F_{0}=\frac{a_{1} a_{6}\left(a_{6}-a_{1}\right) \kappa_{3}+a_{3}^{2}\left(a_{6} \kappa_{1}-a_{1} \kappa_{6}\right)+a_{3}\left(a_{1}^{2} \kappa_{6}-a_{6}^{2} \kappa_{1}\right)}{\left(a_{1}-a_{3}\right)\left(a_{1}-a_{6}\right)\left(a_{3}-a_{6}\right)} \\
& F_{1}=\frac{a_{6}^{2}\left(-\kappa_{1}+\kappa_{3}\right)+a_{3}^{2}\left(\kappa_{1}-\kappa_{6}\right)+a_{1}^{2}\left(-\kappa_{3}+\kappa_{6}\right)}{\left(a_{1}-a_{3}\right)\left(a_{1}-a_{6}\right)\left(a_{3}-a_{6}\right)} \\
& F_{2}=\frac{a_{6}\left(-\kappa_{1}+\kappa_{3}\right)+a_{3}\left(\kappa_{1}-\kappa_{6}\right)+a_{1}\left(-\kappa_{3}+\kappa_{6}\right)}{\left(a_{1}-a_{3}\right)\left(a_{1}-a_{6}\right)\left(a_{3}-a_{6}\right)}
\end{aligned}
$$


Proof. It is proven in the next section that the integrals $F_{0}, F_{1}$ for $g=2$, and the integrals $F_{0}, F_{1}, F_{2}$ for $g=3$, are in involution with respect to the canonical Poisson bracket (and obviously independent). The proposition follows now from the fact that $H_{2,2}^{(r)}=2 F_{0} F_{1}$ for $g=2$ (by $H_{2,2}^{(r)}$ we mean here the part of the Hamiltonian (2.1) related to the points $\left.a_{1}, a_{4}\right)$, and $H_{2,4}^{(r)}=F_{1}^{2}+2 F_{0} F_{2}$ for $g=3$. Hence $H_{2,2}^{(r)}$ (resp., $H_{2,4}^{(r)}$ ) is in involution with the basis integrals.

Remark. It looks like the above vanishing assumptions on the coefficients $p_{i}$ of the equation of the curve do not affect the reduced system. At least, for $g=2$ we have $H_{2,2}^{(r)}=2\left(F_{0}+p_{1} F_{1}\right) F_{1}$ for an arbitrary $p_{1}$, i.e. the basis integrals are the same.

The above listed integrals are related to the universal integrable system introduced in Section 3 (Proposition 3.1). We conclude the section with the following conjecture. The space where a half of the vectors $\alpha_{s}$ is proportional to $(1,0)^{T}$, the remainder to the $(0,1)^{T}$, and $\beta_{s}=0$ for every $s$, is an invariant subspace of the system. The reduced system splits into two independent Hamiltonian completely integrable systems. Each of two reduced systems is given by plugging the corresponding points to the integrals of the universal integrable system of Section [3. The above obtained results prove the conjecture for $g=2,3$.

\section{INTEGRABLE SYSTEM RELATED TO THE LAGRANGE INTERPOLATION POLYNOMIAL}

Let $F(x)=\sum_{i=0}^{n-1} F_{i} x^{i}$ be a polynomial taking values $\kappa_{1}, \ldots, \kappa_{n}$ at the different points $a_{1}, \ldots, a_{n}$ (called nodes in the context of interpolation). Consider its coefficients as functions of the sets of nodes and values:

$$
F_{i}=F_{i}\left(a_{1}, \ldots, a_{n}, \kappa_{1}, \ldots, \kappa_{n}\right), \quad i=0, \ldots, n-1 .
$$

By canonical Poisson bracket we mean here the Poisson bracket in $\mathbb{C}^{2 n}$ equipped with coordinates $a_{1}, \ldots, a_{n}, \kappa_{1}, \ldots, \kappa_{n}$, defined by means the following relations:

$$
\left\{a_{r}, \kappa_{s}\right\}=\delta_{r s}, \quad\left\{a_{r}, a_{s}\right\}=\left\{\kappa_{r}, \kappa_{s}\right\}=0
$$

for all pairs $r, s$, where $\delta_{r s}$ is the Kronecker symbol. The following statement is the main goal of this section.

Proposition 3.1. The functions $F_{i}$ commute with respect to the canonical Poisson bracket.

The proof immediately follows from the following lemma:

Lemma 3.2. There exist rational functions $M_{k}=M_{k}\left(a_{1}, \ldots, a_{n}, \kappa_{1}, \ldots, \kappa_{n}\right)$ such that for any $i=0, \ldots, n-1$

$$
\frac{\partial F_{i}}{\partial a_{k}}=M_{k} \frac{\partial F_{i}}{\partial \kappa_{k}}, \quad k=1, \ldots, n
$$

independently of $i$.

Indeed, by the lemma we obtain

hence

$$
\frac{\partial F_{i}}{\partial a_{k}} \frac{\partial F_{j}}{\partial \kappa_{k}}=M_{k} \frac{\partial F_{i}}{\partial \kappa_{k}} \frac{\partial F_{j}}{\partial \kappa_{k}}
$$

$$
\frac{\partial F_{i}}{\partial a_{k}} \frac{\partial F_{j}}{\partial \kappa_{k}}-\frac{\partial F_{j}}{\partial a_{k}} \frac{\partial F_{i}}{\partial \kappa_{k}}=0,
$$


for all $k=1, \ldots, n$, and

$$
\left\{F_{i}, F_{j}\right\}=\sum_{k=1}^{n}\left(\frac{\partial F_{i}}{\partial a_{k}} \frac{\partial F_{j}}{\partial \kappa_{k}}-\frac{\partial F_{j}}{\partial a_{k}} \frac{\partial F_{i}}{\partial \kappa_{k}}\right)=0 .
$$

Proof of Lemma 3.2, It is obviously sufficient to prove the lemma for $k=1$.

Represent $F$ in the form of Lagrange interpolation polynomial [4]:

$$
F(x)=\sum_{s=1}^{n} \kappa_{s} f_{s}(x)
$$

where

$$
f_{s}(x)=\frac{\left(x-a_{1}\right) \ldots\left(x-a_{s-1}\right)\left(x-a_{s+1}\right) \ldots\left(x-a_{n}\right)}{\left(a_{s}-a_{1}\right) \ldots\left(a_{s}-a_{s-1}\right)\left(a_{s}-a_{s+1}\right) \ldots\left(a_{s}-a_{n}\right)} .
$$

In particular,

hence

$$
f_{1}(x)=\frac{\left(x-a_{2}\right) \ldots\left(x-a_{n}\right)}{\left(a_{1}-a_{2}\right) \ldots\left(a_{1}-a_{n}\right)},
$$

$$
\frac{\partial}{\partial a_{1}} f_{1}(x)=\left(x-a_{2}\right) \ldots\left(x-a_{n}\right) \frac{\partial}{\partial a_{1}} \frac{1}{\left(a_{1}-a_{2}\right) \ldots\left(a_{1}-a_{n}\right)} .
$$

For $s>1$ we have

$$
\frac{\partial}{\partial a_{1}} f_{s}(x)=\frac{\left(x-a_{2}\right) \ldots\left(x-a_{s-1}\right)\left(x-a_{s+1}\right) \ldots\left(x-a_{n}\right)}{\left(a_{s}-a_{2}\right) \ldots\left(a_{s}-a_{s-1}\right)\left(a_{s}-a_{s+1}\right) \ldots\left(a_{s}-a_{n}\right)} \cdot \frac{\partial}{\partial a_{1}} \frac{x-a_{1}}{a_{s}-a_{1}} .
$$

Since

$$
\frac{\partial}{\partial a_{1}} \frac{x-a_{1}}{a_{s}-a_{1}}=\frac{x-a_{s}}{\left(a_{s}-a_{1}\right)^{2}},
$$

the gap in the nominator of the previous expression will get filled up, and we obtain

$$
\frac{\partial}{\partial a_{1}} f_{s}(x)=\frac{\left(x-a_{2}\right) \ldots\left(x-a_{n}\right)}{\left(a_{s}-a_{1}\right)^{2}\left(a_{s}-a_{2}\right) \ldots\left(a_{s}-a_{s-1}\right)\left(a_{s}-a_{s+1}\right) \ldots\left(a_{s}-a_{n}\right)} .
$$

We conclude that

$$
\frac{\partial}{\partial a_{1}} F(x)=M_{1}^{\prime}\left(x-a_{2}\right) \ldots\left(x-a_{n}\right)
$$

where

$$
\begin{aligned}
M_{1}^{\prime} & =\frac{\partial}{\partial a_{1}} \frac{\kappa_{1}}{\left(a_{1}-a_{2}\right) \ldots\left(a_{1}-a_{n}\right)} \\
& +\sum_{s=2}^{n} \frac{\kappa_{s}}{\left(a_{s}-a_{1}\right)^{2}\left(a_{s}-a_{2}\right) \ldots\left(a_{s}-a_{s-1}\right)\left(a_{s}-a_{s+1}\right) \ldots\left(a_{s}-a_{n}\right)} .
\end{aligned}
$$

Further on, by (3.1) we obviously have

$$
\frac{\partial}{\partial \kappa_{1}} F(x)=f_{1}(x)=\frac{\left(x-a_{2}\right) \ldots\left(x-a_{n}\right)}{\left(a_{1}-a_{2}\right) \ldots\left(a_{1}-a_{n}\right)} .
$$

Comparing (3.2) and (3.3), and setting $M_{1}=M_{1}^{\prime}\left(a_{1}-a_{2}\right) \ldots\left(a_{1}-a_{n}\right)$, we conclude that

$$
\frac{\partial}{\partial a_{1}} F(x)=M_{1} \frac{\partial}{\partial \kappa_{1}} F(x) \text {. }
$$

The corresponding relation for the coefficients of the polynomials gives

$$
\frac{\partial F_{i}}{\partial a_{1}}=M_{1} \frac{\partial F_{i}}{\partial \kappa_{1}}
$$


as required.

The proof of the Proposition 3.1 is complete. Observe that Lemma 3.2 can be generalized in the following way.

Lemma 3.3. Let $H$ be an arbitrary polynomial in $F_{0}, F_{1}, \ldots, F_{n-1}$. Then

$$
\frac{\partial H}{\partial a_{k}}=M_{k} \frac{\partial H}{\partial \kappa_{k}}, \quad k=1, \ldots, n .
$$

Proof.

$$
\frac{\partial H}{\partial a_{k}}=\sum_{i=0}^{n-1} \frac{\partial H}{\partial F_{i}} \frac{\partial F_{i}}{\partial a_{k}}=\sum_{i=0}^{n-1} \frac{\partial H}{\partial F_{i}}\left(M_{k} \frac{\partial F_{i}}{\partial \kappa_{k}}\right)=M_{k} \frac{\partial H}{\partial \kappa_{k}} .
$$

Lemma 3.3 is the source of relations of type (2.2). Indeed, by virtue of the canonical system of equations with the Hamiltonian $H$ we have $\dot{a}_{k}=\frac{\partial H}{\partial \kappa_{k}}, \dot{\kappa}_{k}=$ $-\frac{\partial H}{\partial a_{k}}$, and Lemma 3.3 reads as

$$
\dot{\kappa}_{k}=-M_{k} \dot{a}_{k} .
$$

\section{Genus 2. Codes}

(* As the first step we calculate the Lax operator: $\left.{ }^{*}\right)$

$$
\begin{aligned}
& A_{0}:=\left(\begin{array}{ll}
l_{11} & l_{12} \\
l_{21} & l_{22}
\end{array}\right) A_{1}:=\left(\begin{array}{ll}
l_{111} & l_{112} \\
l_{121} & l_{122}
\end{array}\right) \alpha_{1}:=\left(\begin{array}{c}
\alpha_{11} \\
\alpha_{21}
\end{array}\right) \alpha_{2}:=\left(\begin{array}{c}
\alpha_{12} \\
\alpha_{22}
\end{array}\right) \alpha_{3}:=\left(\begin{array}{l}
1 \\
0
\end{array}\right) \\
& \alpha_{4}:=\left(\begin{array}{l}
0 \\
1
\end{array}\right) \beta_{1}:=\left(\begin{array}{l}
\beta_{11} \\
\beta_{21}
\end{array}\right) \beta_{2}:=\left(\begin{array}{l}
\beta_{12} \\
\beta_{22}
\end{array}\right) \beta_{3}:=\left(\begin{array}{c}
0 \\
\beta_{23}
\end{array}\right) \beta_{4}:=\left(\begin{array}{c}
\beta_{14} \\
0
\end{array}\right)
\end{aligned}
$$

$L:=A_{0}+A_{1} x+\alpha_{1}$. Transpose $\left[\beta_{1}\right] *\left(\left(y+b_{1}\right) /\left(x-a_{1}\right)\right)+\alpha_{2}$.Transpose $\left[\beta_{2}\right] *\left(\left(y+b_{2}\right) /\left(x-a_{2}\right)\right)+$

$\alpha_{3}$.Transpose $\left[\beta_{3}\right] *\left(\left(y+b_{3}\right) /\left(x-a_{3}\right)\right)+\alpha_{4}$.Transpose $\left[\beta_{4}\right] *\left(\left(y+b_{4}\right) /\left(x-a_{4}\right)\right)$

(* $L$ is the Lax operator $*$ )

MatrixForm $[L](*$ This results in the expression for $L$. Further on we form the eigenvalue conditions *)

$$
\left(\begin{array}{cc}
l_{11}+\frac{l_{111}}{z^{2}}+\frac{\left(y+b_{1}\right) \alpha_{11} \beta_{11}}{\frac{1}{z^{2}}-a_{1}}+\frac{\left(y+b_{2}\right) \alpha_{12} \beta_{12}}{\frac{1}{z^{2}}-a_{2}} & l_{12}+\frac{l_{112}}{z^{2}}+\frac{\left(y+b_{1}\right) \alpha_{11} \beta_{21}}{\frac{1}{z^{2}}-a_{1}}+\frac{\left(y+b_{2}\right) \alpha_{12} \beta_{22}}{\frac{1}{z^{2}}-a_{2}}+\frac{\left(y+b_{3}\right) \beta_{23}}{\frac{1}{z^{2}}-a_{3}} \\
l_{21}+\frac{l_{121}}{z^{2}}+\frac{\left(y+b_{1}\right) \alpha_{21} \beta_{11}}{\frac{1}{z^{2}}-a_{1}}+\frac{\left(y+b_{2}\right) \alpha_{22} \beta_{12}}{\frac{1}{z^{2}}-a_{2}}+\frac{\left(y+b_{4}\right) \beta_{14}}{\frac{1}{z^{2}}-a_{4}} & l_{22}+\frac{l_{122}}{z^{2}}+\frac{\left(y+b_{1}\right) \alpha_{21} \beta_{21}}{\frac{1}{z^{2}}-a_{1}}+\frac{\left(y+b_{2}\right) \alpha_{22} \beta_{22}}{\frac{1}{z^{2}}-a_{2}}
\end{array}\right)
$$

MatrixForm $\left[\boldsymbol{L} \boldsymbol{\alpha}_{1}\right]\left({ }^{*}\right.$ These 2 commands calculate the left hand $\left.{ }^{*}\right)$

MatrixForm $\left[L \alpha_{2}\right](*$ sides of the subsequent system of equations *)

sol $=$

Solve[ 
$\left\{l_{11}+a_{3} l_{111}+\frac{\left(b_{3}+b_{1}\right) \alpha_{11} \beta_{11}}{a_{3}-a_{1}}+\frac{\left(b_{3}+b_{2}\right) \alpha_{12} \beta_{12}}{a_{3}-a_{2}}==\kappa_{3} \& \& l_{21}+a_{3} l_{121}+\frac{\left(b_{3}+b_{1}\right) \alpha_{21} \beta_{11}}{a_{3}-a_{1}}+\frac{\left(b_{3}+b_{2}\right) \alpha_{22} \beta_{12}}{a_{3}-a_{2}}+\frac{\left(b_{3}+b_{4}\right) \beta_{14}}{a_{3}-a_{4}}==0 \& \&\right.$ $l_{22}+a_{4} l_{122}+\frac{\left(b_{1}+b_{4}\right) \alpha_{21} \beta_{21}}{-a_{1}+a_{4}}+\frac{\left(b_{2}+b_{4}\right) \alpha_{22} \beta_{22}}{-a_{2}+a_{4}}==\kappa_{4} \& \& l_{12}+a_{4} l_{112}+\frac{\left(b_{1}+b_{4}\right) \alpha_{11} \beta_{21}}{-a_{1}+a_{4}}+\frac{\left(b_{2}+b_{4}\right) \alpha_{12} \beta_{22}}{-a_{2}+a_{4}}+\frac{\left(b_{3}+b_{4}\right) \beta_{23}}{-a_{3}+a_{4}}==0 \& \&$ $\alpha_{11}\left(l_{11}+a_{1} l_{111}+\frac{\left(b_{1}+b_{2}\right) \alpha_{12} \beta_{12}}{a_{1}-a_{2}}\right)+\alpha_{21}\left(l_{12}+a_{1} l_{112}+\frac{\left(b_{1}+b_{2}\right) \alpha_{12} \beta_{22}}{a_{1}-a_{2}}+\frac{\left(b_{1}+b_{3}\right) \beta_{23}}{a_{1}-a_{3}}\right)==\kappa_{1} \alpha_{11} \& \&$ $\alpha_{11}\left(l_{21}+a_{1} l_{121}+\frac{\left(b_{1}+b_{2}\right) \alpha_{22} \beta_{12}}{a_{1}-a_{2}}+\frac{\left(b_{1}+b_{4}\right) \beta_{14}}{a_{1}-a_{4}}\right)+\alpha_{21}\left(l_{22}+a_{1} l_{122}+\frac{\left(b_{1}+b_{2}\right) \alpha_{22} \beta_{22}}{a_{1}-a_{2}}\right)==\kappa_{1} \alpha_{21} \& \&$ $\alpha_{12}\left(l_{11}+a_{2} l_{111}+\frac{\left(b_{1}+b_{2}\right) \alpha_{11} \beta_{11}}{-a_{1}+a_{2}}\right)+\alpha_{22}\left(l_{12}+a_{2} l_{112}+\frac{\left(b_{1}+b_{2}\right) \alpha_{11} \beta_{21}}{-a_{1}+a_{2}}+\frac{\left(b_{2}+b_{3}\right) \beta_{23}}{a_{2}-a_{3}}\right)==\kappa_{2} \alpha_{12} \& \&$ $\left.\alpha_{12}\left(l_{21}+a_{2} l_{121}+\frac{\left(b_{1}+b_{2}\right) \alpha_{21} \beta_{11}}{-a_{1}+a_{2}}+\frac{\left(b_{2}+b_{4}\right) \beta_{14}}{a_{2}-a_{4}}\right)+\alpha_{22}\left(l_{22}+a_{2} l_{122}+\frac{\left(b_{1}+b_{2}\right) \alpha_{21} \beta_{21}}{-a_{1}+a_{2}}\right)==\kappa_{2} \alpha_{22}\right\}$, $\left.\left\{l_{11}, l_{12}, l_{21}, l_{22}, l_{111}, l_{112}, l_{121}, l_{122}\right\}\right]$

$x:=1 / z^{\wedge} 2(*$ local parametrization at infinity $*)$

$y=\left(1 / z^{\wedge} 5\right) \operatorname{Sqrt}\left[1+p_{1} z^{\wedge} 2+p_{2} z^{\wedge} 4+p_{3} z^{\wedge} 6+p_{4} z^{\wedge} 8+p_{5} z^{\wedge} 10\right]$

(*Calculating the Hamiltonians as the coefficients of the expansion of Tat infinity : *)

$T=\operatorname{Series}[\operatorname{Tr}[L . L],\{z, 0,-2\}]$

$\left(-1 / z^{\wedge} 3\right) \operatorname{Series}[1 / y,\{z, 0,9\}]\left(* \mathrm{dx} / y\right.$ up to the factor $\left.\mathrm{dz}^{*}\right)$

$\mathrm{H} 2=-$ Residue $\left[(1 / z) * T *\left(-z^{2}+\frac{p_{1} z^{4}}{2}+\left(-\frac{3 p_{1}^{2}}{8}+\frac{p_{2}}{2}\right) z^{6}\right),\{z, 0\}\right]\left(*\right.$ with the differential $\left.z^{\wedge}\{-1\} \mathrm{dx} / y^{*}\right)$

$\left\{\left\{l_{11}, l_{12}, l_{21}, l_{22}, l_{111}, l_{112}, l_{121}, l_{122}\right\}\right\}=\left\{l_{11}, l_{12}, l_{21}, l_{22}, l_{111}, l_{112}, l_{121}, l_{122}\right\} /$. sol

H2 (* by this line we obtain a final expression for H2 taking account of replacements *)

(* The commands below correspond to the Hamiltonian equations with the Hamiltonian H2. The assumptions correspond to the assumptions of the reduction. The zero results prove that assumptions of the reduction are preserved along the trajectories. D $\alpha 11$ and so on denote the time derivatives of the corresponding variables. ${ }^{*}$ )

$\mathrm{DA} 11=\operatorname{Series}\left[D\left[\mathrm{H} 2, \beta_{11}\right] / .\left\{\alpha_{11} \rightarrow 0, \beta_{11} \rightarrow 0, \beta_{21} \rightarrow 0, \beta_{12} \rightarrow 0, \beta_{22} \rightarrow 0, \beta_{13} \rightarrow 0, \beta_{23} \rightarrow 0, \beta_{14} \rightarrow 0\right.\right.$, $\left.\left.\beta_{24} \rightarrow 0\right\},\left\{\alpha_{22}, 0,0\right\}\right] / .\left\{\alpha_{22} \rightarrow 0\right\}$

$\mathrm{D} \beta 11=\operatorname{Series}\left[D\left[\mathrm{H} 2, \alpha_{11}\right] / .\left\{\alpha_{11} \rightarrow 0, \beta_{11} \rightarrow 0, \beta_{21} \rightarrow 0, \beta_{12} \rightarrow 0, \beta_{22} \rightarrow 0, \beta_{13} \rightarrow 0, \beta_{23} \rightarrow 0, \beta_{14} \rightarrow 0\right.\right.$, $\left.\left.\beta_{24} \rightarrow 0\right\},\left\{\alpha_{22}, 0,0\right\}\right] / .\left\{\alpha_{22} \rightarrow 0\right\}$

0

$\mathrm{D} \beta 21=\operatorname{Series}\left[D\left[\mathrm{H} 2, \alpha_{21}\right] / .\left\{\alpha_{11} \rightarrow 0, \beta_{11} \rightarrow 0, \beta_{21} \rightarrow 0, \beta_{12} \rightarrow 0, \beta_{22} \rightarrow 0, \beta_{13} \rightarrow 0, \beta_{23} \rightarrow 0, \beta_{14} \rightarrow 0\right.\right.$, $\left.\left.\beta_{24} \rightarrow 0\right\},\left\{\alpha_{22}, 0,0\right\}\right] / \cdot\left\{\alpha_{22} \rightarrow 0\right\}$ 
$\mathrm{D} \beta 12=-\operatorname{Series}\left[D\left[\mathrm{H} 2, \alpha_{12}\right] / .\left\{\alpha_{11} \rightarrow 0, \beta_{11} \rightarrow 0, \beta_{21} \rightarrow 0, \beta_{12} \rightarrow 0, \beta_{22} \rightarrow 0, \beta_{13} \rightarrow 0, \beta_{23} \rightarrow 0, \beta_{14} \rightarrow 0\right.\right.$, $\left.\left.\beta_{24} \rightarrow 0\right\},\left\{\alpha_{22}, 0,0\right\}\right] / .\left\{\alpha_{22} \rightarrow 0\right\}$

0

$\mathrm{D} \beta 22=-\operatorname{Series}\left[D\left[\mathrm{H} 2, \alpha_{22}\right] / .\left\{\alpha_{11} \rightarrow 0, \beta_{11} \rightarrow 0, \beta_{21} \rightarrow 0, \beta_{12} \rightarrow 0, \beta_{22} \rightarrow 0, \beta_{13} \rightarrow 0, \beta_{23} \rightarrow 0, \beta_{14} \rightarrow 0\right.\right.$, $\left.\left.\beta_{24} \rightarrow 0\right\},\left\{\alpha_{22}, 0,0\right\}\right] / .\left\{\alpha_{22} \rightarrow 0\right\}$

0

$\mathrm{D} \beta 13=-\operatorname{Series}\left[D\left[\mathrm{H} 2, \alpha_{13}\right] / .\left\{\alpha_{11} \rightarrow 0, \beta_{11} \rightarrow 0, \beta_{21} \rightarrow 0, \beta_{12} \rightarrow 0, \beta_{22} \rightarrow 0, \beta_{13} \rightarrow 0, \beta_{23} \rightarrow 0, \beta_{14} \rightarrow 0\right.\right.$, $\left.\left.\beta_{24} \rightarrow 0\right\},\left\{\alpha_{22}, 0,0\right\}\right] / .\left\{\alpha_{22} \rightarrow 0\right\}$

0

$\mathrm{D} \beta 23=-\operatorname{Series}\left[D\left[\mathrm{H} 2, \alpha_{23}\right] / .\left\{\alpha_{11} \rightarrow 0, \beta_{11} \rightarrow 0, \beta_{21} \rightarrow 0, \beta_{12} \rightarrow 0, \beta_{22} \rightarrow 0, \beta_{13} \rightarrow 0, \beta_{23} \rightarrow 0, \beta_{14} \rightarrow 0\right.\right.$, $\left.\left.\beta_{24} \rightarrow 0\right\},\left\{\alpha_{22}, 0,0\right\}\right] / .\left\{\alpha_{22} \rightarrow 0\right\}$

0

$\mathrm{D} \beta 14=-\operatorname{Series}\left[D\left[\mathrm{H} 2, \alpha_{14}\right] / .\left\{\alpha_{11} \rightarrow 0, \beta_{11} \rightarrow 0, \beta_{21} \rightarrow 0, \beta_{12} \rightarrow 0, \beta_{22} \rightarrow 0, \beta_{13} \rightarrow 0, \beta_{23} \rightarrow 0, \beta_{14} \rightarrow 0\right.\right.$, $\left.\left.\beta_{24} \rightarrow 0\right\},\left\{\alpha_{22}, 0,0\right\}\right] / .\left\{\alpha_{22} \rightarrow 0\right\}$

0

$\mathrm{D} \beta 24=-\operatorname{Series}\left[D\left[\mathrm{H} 2, \alpha_{24}\right] / .\left\{\alpha_{11} \rightarrow 0, \beta_{11} \rightarrow 0, \beta_{21} \rightarrow 0, \beta_{12} \rightarrow 0, \beta_{22} \rightarrow 0, \beta_{13} \rightarrow 0, \beta_{23} \rightarrow 0, \beta_{14} \rightarrow 0\right.\right.$, $\left.\left.\beta_{24} \rightarrow 0\right\},\left\{\alpha_{22}, 0,0\right\}\right] / .\left\{\alpha_{22} \rightarrow 0\right\}$

0

$\mathrm{D} \alpha 22=\operatorname{Series}\left[D\left[\mathrm{H} 2, \beta_{22}\right] / .\left\{\alpha_{11} \rightarrow 0, \beta_{11} \rightarrow 0, \beta_{21} \rightarrow 0, \beta_{12} \rightarrow 0, \beta_{22} \rightarrow 0, \beta_{13} \rightarrow 0, \beta_{23} \rightarrow 0, \beta_{14} \rightarrow 0\right.\right.$, $\left.\left.\beta_{24} \rightarrow 0\right\},\left\{\alpha_{22}, 0,0\right\}\right] / .\left\{\alpha_{22} \rightarrow 0\right\}$

0

$\mathrm{D} \alpha 12=\operatorname{Series}\left[D\left[\mathrm{H} 2, \beta_{12}\right] / .\left\{\alpha_{11} \rightarrow 0, \beta_{11} \rightarrow 0, \beta_{21} \rightarrow 0, \beta_{12} \rightarrow 0, \beta_{22} \rightarrow 0, \beta_{13} \rightarrow 0, \beta_{23} \rightarrow 0, \beta_{14} \rightarrow 0\right.\right.$, $\left.\left.\beta_{24} \rightarrow 0\right\},\left\{\alpha_{22}, 0,0\right\}\right] / .\left\{\alpha_{22} \rightarrow 0\right\}$ 
$\operatorname{D} \alpha 21=\operatorname{Series}\left[D\left[\mathrm{H} 2, \beta_{21}\right] / .\left\{\alpha_{11} \rightarrow 0, \beta_{11} \rightarrow 0, \beta_{21} \rightarrow 0, \beta_{12} \rightarrow 0, \beta_{22} \rightarrow 0, \beta_{13} \rightarrow 0, \beta_{23} \rightarrow 0, \beta_{14} \rightarrow 0\right.\right.$, $\left.\left.\beta_{24} \rightarrow 0\right\},\left\{\alpha_{22}, 0,0\right\}\right] / \cdot\left\{\alpha_{22} \rightarrow 0\right\}$

$\frac{2\left(b_{1}+b_{4}\right)\left(a_{1} \alpha_{21} \kappa_{1}+a_{4} \alpha_{21} \kappa_{1}-2 a_{1} \alpha_{21} \kappa_{4}\right)}{\left(a_{1}-a_{4}\right)^{3}}$

$\operatorname{Da} 1=\operatorname{Series}\left[D\left[\mathrm{H} 2, \kappa_{1}\right] / .\left\{\alpha_{11} \rightarrow 0, \beta_{11} \rightarrow 0, \beta_{21} \rightarrow 0, \beta_{12} \rightarrow 0, \beta_{22} \rightarrow 0, \beta_{13} \rightarrow 0, \beta_{23} \rightarrow 0, \beta_{14} \rightarrow 0\right.\right.$, $\left.\left.\beta_{24} \rightarrow 0\right\},\left\{\alpha_{22}, 0,0\right\}\right] / .\left\{\alpha_{22} \rightarrow 0\right\}$

$\frac{2\left(-2 a_{4} \kappa_{1}+a_{1} \kappa_{4}+a_{4} \kappa_{4}\right)}{\left(a_{1}-a_{4}\right)^{2}}$

$\operatorname{Da} 2=\operatorname{Series}\left[D\left[\mathrm{H} 2, \kappa_{2}\right] / .\left\{\alpha_{11} \rightarrow 0, \beta_{11} \rightarrow 0, \beta_{21} \rightarrow 0, \beta_{12} \rightarrow 0, \beta_{22} \rightarrow 0, \beta_{13} \rightarrow 0, \beta_{23} \rightarrow 0, \beta_{14} \rightarrow 0\right.\right.$, $\left.\left.\beta_{24} \rightarrow 0\right\},\left\{\alpha_{22}, 0,0\right\}\right] / .\left\{\alpha_{22} \rightarrow 0\right\}$

$\frac{2\left(-2 a_{3} \kappa_{2}+a_{2} \kappa_{3}+a_{3} \kappa_{3}\right)}{\left(a_{2}-a_{3}\right)^{2}}$

$\operatorname{Da} 3=\operatorname{Series}\left[D\left[\mathrm{H} 2, \kappa_{3}\right] / .\left\{\alpha_{11} \rightarrow 0, \beta_{11} \rightarrow 0, \beta_{21} \rightarrow 0, \beta_{12} \rightarrow 0, \beta_{22} \rightarrow 0, \beta_{13} \rightarrow 0, \beta_{23} \rightarrow 0, \beta_{14} \rightarrow 0\right.\right.$, $\left.\left.\beta_{24} \rightarrow 0\right\},\left\{\alpha_{22}, 0,0\right\}\right] / .\left\{\alpha_{22} \rightarrow 0\right\}$

$\frac{2\left(a_{2} \kappa_{2}+a_{3} \kappa_{2}-2 a_{2} \kappa_{3}\right)}{\left(a_{2}-a_{3}\right)^{2}}$

$\operatorname{Da} 4=\operatorname{Series}\left[D\left[\mathrm{H} 2, \kappa_{4}\right] / .\left\{\alpha_{11} \rightarrow 0, \beta_{11} \rightarrow 0, \beta_{21} \rightarrow 0, \beta_{12} \rightarrow 0, \beta_{22} \rightarrow 0, \beta_{13} \rightarrow 0, \beta_{23} \rightarrow 0, \beta_{14} \rightarrow 0\right.\right.$, $\left.\left.\beta_{24} \rightarrow 0\right\},\left\{\alpha_{22}, 0,0\right\}\right] / .\left\{\alpha_{22} \rightarrow 0\right\}$ $\frac{2\left(a_{1} \kappa_{1}+a_{4} \kappa_{1}-2 a_{1} \kappa_{4}\right)}{\left(a_{1}-a_{4}\right)^{2}}$

$\mathrm{D} \kappa 1=\operatorname{Series}\left[-D\left[\mathrm{H} 2, a_{1}\right] / .\left\{\alpha_{11} \rightarrow 0, \beta_{11} \rightarrow 0, \beta_{21} \rightarrow 0, \beta_{12} \rightarrow 0, \beta_{22} \rightarrow 0, \beta_{13} \rightarrow 0, \beta_{23} \rightarrow 0, \beta_{14} \rightarrow 0\right.\right.$, $\left.\left.\beta_{24} \rightarrow 0\right\},\left\{\alpha_{22}, 0,0\right\}\right] / .\left\{\alpha_{22} \rightarrow 0\right\}$

$\frac{2\left(\kappa_{1}-\kappa_{4}\right)\left(-2 a_{4} \kappa_{1}+a_{1} \kappa_{4}+a_{4} \kappa_{4}\right)}{\left(a_{1}-a_{4}\right)^{3}}$

$\mathrm{D} \kappa 2=\operatorname{Series}\left[-D\left[\mathrm{H} 2, a_{2}\right] / .\left\{\alpha_{11} \rightarrow 0, \beta_{11} \rightarrow 0, \beta_{21} \rightarrow 0, \beta_{12} \rightarrow 0, \beta_{22} \rightarrow 0, \beta_{13} \rightarrow 0, \beta_{23} \rightarrow 0, \beta_{14} \rightarrow 0\right.\right.$, $\left.\left.\beta_{24} \rightarrow 0\right\},\left\{\alpha_{22}, 0,0\right\}\right] / .\left\{\alpha_{22} \rightarrow 0\right\}$

$\frac{2\left(\kappa_{2}-\kappa_{3}\right)\left(-2 a_{3} \kappa_{2}+a_{2} \kappa_{3}+a_{3} \kappa_{3}\right)}{\left(a_{2}-a_{3}\right)^{3}}$

$\mathrm{D} \kappa 3=\operatorname{Series}\left[-D\left[\mathrm{H} 2, a_{3}\right] / .\left\{\alpha_{11} \rightarrow 0, \beta_{11} \rightarrow 0, \beta_{21} \rightarrow 0, \beta_{12} \rightarrow 0, \beta_{22} \rightarrow 0, \beta_{13} \rightarrow 0, \beta_{23} \rightarrow 0, \beta_{14} \rightarrow 0\right.\right.$, $\left.\left.\beta_{24} \rightarrow 0\right\},\left\{\alpha_{22}, 0,0\right\}\right] / .\left\{\alpha_{22} \rightarrow 0\right\}$ 
$\mathrm{D} \kappa 4=\operatorname{Series}\left[-D\left[\mathrm{H} 2, a_{4}\right] / .\left\{\alpha_{11} \rightarrow 0, \beta_{11} \rightarrow 0, \beta_{21} \rightarrow 0, \beta_{12} \rightarrow 0, \beta_{22} \rightarrow 0, \beta_{13} \rightarrow 0, \beta_{23} \rightarrow 0, \beta_{14} \rightarrow 0\right.\right.$,

$\left.\left.\beta_{24} \rightarrow 0\right\},\left\{\alpha_{22}, 0,0\right\}\right] / \cdot\left\{\alpha_{22} \rightarrow 0\right\}$

$-\frac{2\left(\kappa_{1}-\kappa_{4}\right)\left(a_{1} \kappa_{1}+a_{4} \kappa_{1}-2 a_{1} \kappa_{4}\right)}{\left(-a_{1}+a_{4}\right)^{3}}$

H2r $=$ Simplify[Series[H2/. $\left\{\alpha_{11} \rightarrow 0, \beta_{11} \rightarrow 0, \beta_{21} \rightarrow 0, \beta_{12} \rightarrow 0, \beta_{22} \rightarrow 0, \beta_{13} \rightarrow 0, \beta_{23} \rightarrow 0, \beta_{14} \rightarrow 0\right.$,

$\left.\left.\left.\beta_{24} \rightarrow 0\right\},\left\{\alpha_{22}, 0,0\right\}\right] / .\left\{\alpha_{22} \rightarrow 0\right\}\right]$

$-\frac{1}{\left(a_{2}-a_{3}\right)^{2}\left(a_{1}-a_{4}\right)^{2}} 2\left(a_{2}^{2}\left(\kappa_{1}-\kappa_{4}\right)\left(a_{4} \kappa_{1}-a_{1} \kappa_{4}\right)+a_{2}\left(-\left(a_{1}-a_{4}\right)^{2}\left(\kappa_{2}-\kappa_{3}\right) \kappa_{3}-2 a_{3}\left(\kappa_{1}-\right.\right.\right.$

$\left.\left.\left.\kappa_{4}\right)\left(a_{4} \kappa_{1}-a_{1} \kappa_{4}\right)\right)+a_{3}\left(\left(a_{1}-a_{4}\right)^{2} \kappa_{2}\left(\kappa_{2}-\kappa_{3}\right)+a_{3}\left(\kappa_{1}-\kappa_{4}\right)\left(a_{4} \kappa_{1}-a_{1} \kappa_{4}\right)\right)\right)$

(*It is equal to $2\left(\kappa_{1}-\kappa_{4}\right)\left(a_{4} \kappa_{1}-a_{1} \kappa_{4}\right) /\left(a_{1}-a_{4}\right)^{2}+2\left(\kappa_{2}-\kappa_{3}\right)\left(a_{3} \kappa_{2}-a_{2} \kappa_{3}\right) /\left(a_{2}-a_{3}\right)^{2}$ as in Proposition $\left.1 *\right)$

\section{Genus 3. Codes}

$A_{0}:=\left(\begin{array}{ll}l_{11} & l_{12} \\ l_{21} & l_{22}\end{array}\right) A_{1}:=\left(\begin{array}{ll}l_{111} & l_{112} \\ l_{121} & l_{122}\end{array}\right) A_{2}:=\left(\begin{array}{ll}l_{211} & l_{212} \\ l_{221} & l_{222}\end{array}\right)$

$\alpha_{1}:=\left(\begin{array}{c}\alpha_{11} \\ \alpha_{21}\end{array}\right) \alpha_{2}:=\left(\begin{array}{c}\alpha_{12} \\ \alpha_{22}\end{array}\right) \alpha_{3}:=\left(\begin{array}{l}\alpha_{13} \\ \alpha_{23}\end{array}\right) \alpha_{4}:=\left(\begin{array}{l}\alpha_{14} \\ \alpha_{24}\end{array}\right) \alpha_{5}:=\left(\begin{array}{l}1 \\ 0\end{array}\right) \alpha_{6}:=\left(\begin{array}{l}0 \\ 1\end{array}\right)$

$\beta_{1}:=\left(\begin{array}{c}\beta_{11} \\ \beta_{21}\end{array}\right) \beta_{2}:=\left(\begin{array}{c}\beta_{12} \\ \beta_{22}\end{array}\right) \beta_{3}:=\left(\begin{array}{c}\beta_{13} \\ \beta_{23}\end{array}\right) \beta_{4}:=\left(\begin{array}{c}\beta_{14} \\ \beta_{24}\end{array}\right) \beta_{5}:=\left(\begin{array}{c}0 \\ \beta_{25}\end{array}\right) \beta_{6}:=\left(\begin{array}{c}\beta_{16} \\ 0\end{array}\right)$

$\left({ }^{*} L\right.$ is the Lax operator: $\left.{ }^{*}\right)$

$L:=A_{0}+A_{1} x+A_{2} x^{2}+\alpha_{1}$.Transpose $\left[\beta_{1}\right] *\left(\left(y+b_{1}\right) /\left(x-a_{1}\right)\right)+\alpha_{2}$.Transpose $\left[\beta_{2}\right] *\left(\left(y+b_{2}\right) /\left(x-a_{2}\right)\right)+$

$\alpha_{3}$.Transpose $\left[\beta_{3}\right] *\left(\left(y+b_{3}\right) /\left(x-a_{3}\right)\right)+\alpha_{4}$. Transpose $\left[\beta_{4}\right] *\left(\left(y+b_{4}\right) /\left(x-a_{4}\right)\right)+\alpha_{5}$. Transpose $\left[\beta_{5}\right] *\left(\left(y+b_{5}\right) /\left(x-a_{5}\right)\right)+$

$\alpha_{6}$. Transpose $\left[\beta_{6}\right] *\left(\left(y+b_{6}\right) /\left(x-a_{6}\right)\right)$

$\left({ }^{*}\right.$ Calculating the Hamiltonian : *)

$x:=1 / z^{\wedge} 2$

$y=\left(1 / z^{\wedge} 7\right) \operatorname{Sqrt}\left[1+p_{1} z^{\wedge} 2+p_{2} z^{\wedge} 4+p_{3} z^{\wedge} 6+p_{4} z^{\wedge} 8+p_{5} z^{\wedge} 10+p_{6} z^{\wedge} 12+p_{7} z^{\wedge} 14\right]$

(* at the infinity $1 / y \sim z^{7}, z^{-1} \mathrm{dx} / y \sim z^{3} \mathrm{dz}$, hence $\operatorname{Tr}[$ L.L $]$ should be calculated up to $z^{-4}{ }^{*}$ )

$T=\operatorname{Series}[\operatorname{Tr}[L . L],\{z, 0,-4\}]$

$\left({ }^{*} \operatorname{Tr}[L . L] \sim z^{-10}, z^{-1} \operatorname{Tr}[L . L] \sim z^{-11}\right.$, hence $\mathrm{dx} / y$ should be calculated up to $\left.z^{10} *\right)$ 
$\left(-1 / z^{\wedge} 3\right) \operatorname{Series}[1 / y,\{z, 0,13\}]\left(* \mathrm{dx} / y\right.$ up to the factor $\left.\mathrm{dz}{ }^{*}\right)$

$\left({ }^{*}\right.$ For $p_{1}=p_{2}=p_{3}=0$ the Hamiltonian at $z^{-5}$ gives trivial equations. $\left.{ }^{*}\right)$

(* The following Hamiltonian is the Hamiltonian at $z^{-4}\left(\right.$ for $\left.\left.p_{1}=p_{2}=p_{3}=0\right):{ }^{*}\right)$

$\mathrm{H} 2=-\operatorname{Residue}\left[(1 / z) * T *\left(-z^{4}+\frac{p_{1} z^{6}}{2}+\left(-\frac{3 p_{1}^{2}}{8}+\frac{p_{2}}{2}\right) z^{8}+\frac{1}{16}\left(5 p_{1}^{3}-12 p_{1} p_{2}+8 p_{3}\right) z^{10}\right),\{z, 0\}\right]$

(* with the differential $z^{-1} \mathrm{dx} / y$. Below $p_{1}=p_{2}=p_{3}=0 *$ )

(* First part of the equations out of eigenvalue conditions

(to generate the left hand sides of those equations one makes use of commands of the type MatrixForm[L $\left.\alpha_{i}\right]$ like it has been done for genus $=2$ ) : $*$ )

sol $=$

Solve $\left[\left\{l_{11}+a_{5} l_{111}+a_{5}^{2} l_{211}+\frac{\left(b_{5}+b_{1}\right) \alpha_{11} \beta_{11}}{a_{5}-a_{1}}+\frac{\left(b_{5}+b_{2}\right) \alpha_{12} \beta_{12}}{a_{5}-a_{2}}+\frac{\left(b_{5}+b_{3}\right) \alpha_{13} \beta_{13}}{a_{5}-a_{3}}+\frac{\left(b_{5}+b_{4}\right) \alpha_{14} \beta_{14}}{a_{5}-a_{4}}==\kappa 5 \& \&\right.\right.$..

$l_{12}+a_{6} l_{112}+a_{6}{ }^{2} l_{212}+\frac{\left(b_{1}+b_{6}\right) \alpha_{11} \beta_{21}}{-a_{1}+a_{6}}+\frac{\left(b_{2}+b_{6}\right) \alpha_{12} \beta_{22}}{-a_{2}+a_{6}}+\frac{\left(b_{3}+b_{6}\right) \alpha_{13} \beta_{23}}{-a_{3}+a_{6}}+\frac{\left(b_{4}+b_{6}\right) \alpha_{14} \beta_{24}}{-a_{4}+a_{6}}+\frac{\left(b_{5}+b_{6}\right) \beta_{25}}{-a_{5}+a_{6}}==0 \& \&$

$\alpha_{11}\left(l_{11}+a_{1} l_{111}+a_{1}^{2} l_{211}+\frac{\left(b_{1}+b_{2}\right) \alpha_{12} \beta_{12}}{a_{1}-a_{2}}+\frac{\left(b_{1}+b_{3}\right) \alpha_{13} \beta_{13}}{a_{1}-a_{3}}+\frac{\left(b_{1}+b_{4}\right) \alpha_{14} \beta_{14}}{a_{1}-a_{4}}\right)+$

$\alpha_{21}\left(l_{12}+a_{1} l_{112}+a_{1}^{2} l_{212}+\frac{\left(b_{1}+b_{2}\right) \alpha_{12} \beta_{22}}{a_{1}-a_{2}}+\frac{\left(b_{1}+b_{3}\right) \alpha_{13} \beta_{23}}{a_{1}-a_{3}}+\frac{\left(b_{1}+b_{4}\right) \alpha_{14} \beta_{24}}{a_{1}-a_{4}}+\frac{\left(b_{1}+b_{5}\right) \beta_{25}}{a_{1}-a_{5}}\right)==\kappa_{1} \alpha_{11} \& \&$

$\alpha_{12}\left(l_{11}+a_{2} l_{111}+a_{2}^{2} l_{211}+\frac{\left(b_{1}+b_{2}\right) \alpha_{11} \beta_{11}}{-a_{1}+a_{2}}+\frac{\left(b_{3}+b_{2}\right) \alpha_{13} \beta_{13}}{-a_{3}+a_{2}}+\frac{\left(b_{4}+b_{2}\right) \alpha_{14} \beta_{14}}{-a_{4}+a_{2}}\right)+$

$\alpha_{22}\left(l_{12}+a_{2} l_{112}+a_{2}^{2} l_{212}+\frac{\left(b_{1}+b_{2}\right) \alpha_{11} \beta_{21}}{-a_{1}+a_{2}}+\frac{\left(b_{3}+b_{2}\right) \alpha_{13} \beta_{23}}{-a_{3}+a_{2}}+\frac{\left(b_{4}+b_{2}\right) \alpha_{14} \beta_{24}}{-a_{4}+a_{2}}+\frac{\left(b_{2}+b_{5}\right) \beta_{25}}{a_{2}-a_{5}}\right)==\kappa_{2} \alpha_{12} \& \&$

$\alpha_{13}\left(l_{11}+a_{3} l_{111}+a_{3}^{2} l_{211}+\frac{\left(b_{1}+b_{3}\right) \alpha_{11} \beta_{11}}{-a_{1}+a_{3}}+\frac{\left(b_{3}+b_{2}\right) \alpha_{12} \beta_{12}}{-a_{2}+a_{3}}+\frac{\left(b_{4}+b_{3}\right) \alpha_{14} \beta_{14}}{-a_{4}+a_{3}}\right)+$

$\alpha_{23}\left(l_{12}+a_{3} l_{112}+a_{3}^{2} l_{212}+\frac{\left(b_{1}+b_{3}\right) \alpha_{11} \beta_{21}}{-a_{1}+a_{3}}+\frac{\left(b_{3}+b_{2}\right) \alpha_{12} \beta_{22}}{-a_{2}+a_{3}}+\frac{\left(b_{4}+b_{3}\right) \alpha_{14} \beta_{24}}{-a_{4}+a_{3}}+\frac{\left(b_{3}+b_{5}\right) \beta_{25}}{a_{3}-a_{5}}\right)==\kappa_{3} \alpha_{13} \& \&$

$\alpha_{14}\left(l_{11}+a_{4} l_{111}+a_{4}^{2} l_{211}+\frac{\left(b_{1}+b_{4}\right) \alpha_{11} \beta_{11}}{-a_{1}+a_{4}}+\frac{\left(b_{4}+b_{2}\right) \alpha_{12} \beta_{12}}{-a_{2}+a_{4}}+\frac{\left(b_{4}+b_{3}\right) \alpha_{13} \beta_{13}}{-a_{3}+a_{4}}\right)+$

$\left.. \alpha_{24}\left(l_{12}+a_{4} l_{112}+a_{4}^{2} l_{212}+\frac{\left(b_{1}+b_{4}\right) \alpha_{11} \beta_{21}}{-a_{1}+a_{4}}+\frac{\left(b_{4}+b_{2}\right) \alpha_{12} \beta_{22}}{-a_{2}+a_{4}}+\frac{\left(b_{4}+b_{3}\right) \alpha_{13} \beta_{23}}{-a_{3}+a_{4}}+\frac{\left(b_{4}+b_{5}\right) \beta_{25}}{a_{4}-a_{5}}\right)==\kappa_{4} \alpha_{14}\right\}$,

. $\left.\left\{l_{11}, l_{12}, l_{111}, l_{112}, l_{211}, l_{212}\right\}\right]$

$\left\{\left\{l_{11}, l_{12}, l_{111}, l_{112}, l_{211}, l_{212}\right\}\right\}=\left\{l_{11}, l_{12}, l_{111}, l_{112}, l_{211}, l_{212}\right\} /$. sol

(* Second part of equations out of eigenvalue conditions: *)

sol $=$

Solve $\left[\left\{l_{21}+a_{5} l_{121}+a_{5}^{2} l_{221}+\frac{\left(b_{5}+b_{1}\right) \alpha_{21} \beta_{11}}{a_{5}-a_{1}}+\frac{\left(b_{5}+b_{2}\right) \alpha_{22} \beta_{12}}{a_{5}-a_{2}}+\frac{\left(b_{5}+b_{3}\right) \alpha_{23} \beta_{13}}{a_{5}-a_{3}}+\frac{\left(b_{5}+b_{4}\right) \alpha_{24} \beta_{14}}{a_{5}-a_{4}}+\frac{\left(b_{5}+b_{6}\right) \beta_{16}}{a_{5}-a_{6}}==0 \& \&\right.\right.$.

$l_{22}+a_{6} l_{122}+a_{6}^{2} l_{222}+\frac{\left(b_{1}+b_{6}\right) \alpha_{21} \beta_{21}}{-a_{1}+a_{6}}+\frac{\left(b_{2}+b_{6}\right) \alpha_{22} \beta_{22}}{-a_{2}+a_{6}}+\frac{\left(b_{3}+b_{6}\right) \alpha_{23} \beta_{23}}{-a_{3}+a_{6}}+\frac{\left(b_{4}+b_{6}\right) \alpha_{24} \beta_{24}}{-a_{4}+a_{6}}==\kappa_{6} \& \&$

$\alpha_{11}\left(l_{21}+a_{1} l_{121}+a_{1}^{2} l_{221}+\frac{\left(b_{1}+b_{2}\right) \alpha_{22} \beta_{12}}{a_{1}-a_{2}}+\frac{\left(b_{1}+b_{3}\right) \alpha_{23} \beta_{13}}{a_{1}-a_{3}}+\frac{\left(b_{1}+b_{4}\right) \alpha_{24} \beta_{14}}{a_{1}-a_{4}}+\frac{\left(b_{1}+b_{6}\right) \beta_{16}}{a_{1}-a_{6}}\right)+$

$\alpha_{21}\left(l_{22}+a_{1} l_{122}+a_{1}^{2} l_{222}+\frac{\left(b_{1}+b_{2}\right) \alpha_{22} \beta_{22}}{a_{1}-a_{2}}+\frac{\left(b_{1}+b_{3}\right) \alpha_{23} \beta_{23}}{a_{1}-a_{3}}+\frac{\left(b_{1}+b_{4}\right) \alpha_{24} \beta_{24}}{a_{1}-a_{4}}\right)==\kappa_{1} \alpha_{21} \& \&$

$\alpha_{12}\left(l_{21}+a_{2} l_{121}+a_{2}^{2} l_{221}+\frac{\left(b_{1}+b_{2}\right) \alpha_{21} \beta_{11}}{-a_{1}+a_{2}}+\frac{\left(b_{3}+b_{2}\right) \alpha_{23} \beta_{13}}{-a_{3}+a_{2}}+\frac{\left(b_{4}+b_{2}\right) \alpha_{24} \beta_{14}}{-a_{4}+a_{2}}+\frac{\left(b_{2}+b_{6}\right) \beta_{16}}{a_{2}-a_{6}}\right)+$

$\alpha_{22}\left(l_{22}+a_{2} l_{122}+a_{2}^{2} l_{222}+\frac{\left(b_{1}+b_{2}\right) \alpha_{21} \beta_{21}}{-a_{1}+a_{2}}+\frac{\left(b_{3}+b_{2}\right) \alpha_{23} \beta_{23}}{-a_{3}+a_{2}}+\frac{\left(b_{4}+b_{2}\right) \alpha_{24} \beta_{24}}{-a_{4}+a_{2}}\right)==\kappa_{2} \alpha_{22} \& \&$ 


$$
\begin{aligned}
& \alpha_{13}\left(l_{21}+a_{3} l_{121}+a_{3}{ }^{2} l_{221}+\frac{\left(b_{1}+b_{3}\right) \alpha_{21} \beta_{11}}{-a_{1}+a_{3}}+\frac{\left(b_{3}+b_{2}\right) \alpha_{22} \beta_{12}}{-a_{2}+a_{3}}+\frac{\left(b_{4}+b_{3}\right) \alpha_{24} \beta_{14}}{-a_{4}+a_{3}}+\frac{\left(b_{3}+b_{6}\right) \beta_{16}}{a_{3}-a_{6}}\right)+ \\
& \alpha_{23}\left(l_{22}+a_{3} l_{122}+a_{3}{ }^{2} l_{222}+\frac{\left(b_{1}+b_{3}\right) \alpha_{21} \beta_{21}}{-a_{1}+a_{3}}+\frac{\left(b_{3}+b_{2}\right) \alpha_{22} \beta_{22}}{-a_{2}+a_{3}}+\frac{\left(b_{4}+b_{3}\right) \alpha_{24} \beta_{24}}{-a_{4}+a_{3}}\right)==\kappa_{3} \alpha_{23} \& \& \\
& \alpha_{14}\left(l_{21}+a_{4} l_{121}+a_{4}{ }^{2} l_{221}+\frac{\left(b_{1}+b_{4}\right) \alpha_{21} \beta_{11}}{-a_{1}+a_{4}}+\frac{\left(b_{4}+b_{2}\right) \alpha_{22} \beta_{12}}{-a_{2}+a_{4}}+\frac{\left(b_{4}+b_{3}\right) \alpha_{23} \beta_{13}}{-a_{3}+a_{4}}+\frac{\left(b_{4}+b_{6}\right) \beta_{16}}{a_{4}-a_{6}}\right)+ \\
& \left.\left.. . \alpha_{24}\left(l_{22}+a_{4} l_{122}+a_{4}{ }^{2} l_{222}+\frac{\left(b_{1}+b_{4}\right) \alpha_{21} \beta_{21}}{-a_{1}+a_{4}}+\frac{\left(b_{4}+b_{2}\right) \alpha_{22} \beta_{22}}{-a_{2}+a_{4}}+\frac{\left(b_{4}+b_{3}\right) \alpha_{23} \beta_{23}}{-a_{3}+a_{4}}\right)==\kappa_{4} \alpha_{24}\right\},\left\{l_{21}, l_{22}, l_{121}, l_{122}, l_{221}, l_{222}\right\}\right] \\
& \left\{\left\{l_{21}, l_{22}, l_{121}, l_{122}, l_{221}, l_{222}\right\}\right\}=\left\{l_{21}, l_{22}, l_{121}, l_{122}, l_{221}, l_{222}\right\} / . \mathrm{sol}
\end{aligned}
$$

(* It is recommended to collect and keep the results of the foregoing replacements for $l_{\mathrm{ij}}$,

$l_{\mathrm{ijk}}$ in a separate file because solution of the system takes quite a lot time ${ }^{*}$ )

$\mathrm{H} 2$ (*calculating the Hamiltonian taking account of the replacements for $l_{\mathrm{ij}}, l_{\mathrm{ijk}}{ }^{*}$ )

(* Next we check admissibility of the reduction: *)

$$
\begin{aligned}
& \text { DA11 = Simplify[Series }\left[D\left[\mathrm{H} 2, \beta_{11}\right] / \cdot\left\{\alpha_{11} \rightarrow 0, \alpha_{13} \rightarrow 0, \alpha_{24} \rightarrow 0, \beta_{11} \rightarrow 0, \beta_{21} \rightarrow 0, \beta_{12} \rightarrow 0, \beta_{22} \rightarrow 0, \beta_{13} \rightarrow 0\right.\right. \\
& \left.\left.\left.\beta_{23} \rightarrow 0, \beta_{14} \rightarrow 0, \beta_{24} \rightarrow 0, \beta_{25} \rightarrow 0, \beta_{16} \rightarrow 0\right\},\left\{\alpha_{22}, 0,0\right\}\right] / \cdot\left\{\alpha_{22} \rightarrow 0\right\}\right]
\end{aligned}
$$$$
0
$$$$
\text { DA22 = Simplify[Series }\left[D\left[\mathrm{H} 2, \beta_{22}\right] / \cdot\left\{\alpha_{11} \rightarrow 0, \alpha_{13} \rightarrow 0, \alpha_{24} \rightarrow 0, \beta_{11} \rightarrow 0, \beta_{21} \rightarrow 0, \beta_{12} \rightarrow 0, \beta_{22} \rightarrow 0, \beta_{13} \rightarrow 0\right. \text {, }\right.
$$$$
\left.\left.\left.\beta_{23} \rightarrow 0, \beta_{14} \rightarrow 0, \beta_{24} \rightarrow 0, \beta_{25} \rightarrow 0, \beta_{16} \rightarrow 0\right\},\left\{\alpha_{22}, 0,0\right\}\right] / .\left\{\alpha_{22} \rightarrow 0\right\}\right]
$$

0

$\mathrm{DA} 13=\operatorname{Simplify}\left[\operatorname{Series}\left[D\left[\mathrm{H} 2, \beta_{13}\right] / .\left\{\alpha_{11} \rightarrow 0, \alpha_{13} \rightarrow 0, \alpha_{24} \rightarrow 0, \beta_{11} \rightarrow 0, \beta_{21} \rightarrow 0, \beta_{12} \rightarrow 0, \beta_{22} \rightarrow 0, \beta_{13} \rightarrow 0\right.\right.\right.$ $\left.\left.\left.\beta_{23} \rightarrow 0, \beta_{14} \rightarrow 0, \beta_{24} \rightarrow 0, \beta_{25} \rightarrow 0, \beta_{16} \rightarrow 0\right\},\left\{\alpha_{22}, 0,0\right\}\right] / .\left\{\alpha_{22} \rightarrow 0\right\}\right]$

0

$\mathrm{DA} 24=\operatorname{Simplify}\left[\operatorname{Series}\left[D\left[\mathrm{H} 2, \beta_{24}\right] / .\left\{\alpha_{11} \rightarrow 0, \alpha_{13} \rightarrow 0, \alpha_{24} \rightarrow 0, \beta_{11} \rightarrow 0, \beta_{21} \rightarrow 0, \beta_{12} \rightarrow 0, \beta_{22} \rightarrow 0, \beta_{13} \rightarrow 0\right.\right.\right.$ $\left.\left.\left.\beta_{23} \rightarrow 0, \beta_{14} \rightarrow 0, \beta_{24} \rightarrow 0, \beta_{25} \rightarrow 0, \beta_{16} \rightarrow 0\right\},\left\{\alpha_{22}, 0,0\right\}\right] / .\left\{\alpha_{22} \rightarrow 0\right\}\right]$

0

$$
\begin{aligned}
& \mathrm{DB} 11=\text { Simplify[Series }\left[D\left[\mathrm{H} 2, \alpha_{11}\right] / .\left\{\alpha_{11} \rightarrow 0, \alpha_{13} \rightarrow 0, \alpha_{24} \rightarrow 0, \beta_{11} \rightarrow 0, \beta_{21} \rightarrow 0, \beta_{12} \rightarrow 0, \beta_{22} \rightarrow 0, \beta_{13} \rightarrow 0\right.\right. \\
& \left.\left.\left.\beta_{23} \rightarrow 0, \beta_{14} \rightarrow 0, \beta_{24} \rightarrow 0, \beta_{25} \rightarrow 0, \beta_{16} \rightarrow 0\right\},\left\{\alpha_{22}, 0,0\right\}\right] / .\left\{\alpha_{22} \rightarrow 0\right\}\right]
\end{aligned}
$$

0

$$
\begin{aligned}
& \text { DB21 = Simplify[Series }\left[D\left[\mathrm{H} 2, \alpha_{21}\right] / .\left\{\alpha_{11} \rightarrow 0, \alpha_{13} \rightarrow 0, \alpha_{24} \rightarrow 0, \beta_{11} \rightarrow 0, \beta_{21} \rightarrow 0, \beta_{12} \rightarrow 0, \beta_{22} \rightarrow 0, \beta_{13} \rightarrow 0\right.\right. \\
& \left.\left.\left.\beta_{23} \rightarrow 0, \beta_{14} \rightarrow 0, \beta_{24} \rightarrow 0, \beta_{25} \rightarrow 0, \beta_{16} \rightarrow 0\right\},\left\{\alpha_{22}, 0,0\right\}\right] / .\left\{\alpha_{22} \rightarrow 0\right\}\right]
\end{aligned}
$$


0

$\mathrm{DB} 12=\operatorname{Simplify}\left[\operatorname{Series}\left[D\left[\mathrm{H} 2, \alpha_{12}\right] / .\left\{\alpha_{11} \rightarrow 0, \alpha_{13} \rightarrow 0, \alpha_{24} \rightarrow 0, \beta_{11} \rightarrow 0, \beta_{21} \rightarrow 0, \beta_{12} \rightarrow 0, \beta_{22} \rightarrow 0, \beta_{13} \rightarrow 0\right.\right.\right.$, $\left.\left.\left.\beta_{23} \rightarrow 0, \beta_{14} \rightarrow 0, \beta_{24} \rightarrow 0, \beta_{25} \rightarrow 0, \beta_{16} \rightarrow 0\right\},\left\{\alpha_{22}, 0,0\right\}\right] / .\left\{\alpha_{22} \rightarrow 0\right\}\right]$

0

$\mathrm{DB} 22=\operatorname{Simplify}\left[\operatorname{Series}\left[D\left[\mathrm{H} 2, \alpha_{22}\right] / .\left\{\alpha_{11} \rightarrow 0, \alpha_{13} \rightarrow 0, \alpha_{24} \rightarrow 0, \beta_{11} \rightarrow 0, \beta_{21} \rightarrow 0, \beta_{12} \rightarrow 0, \beta_{22} \rightarrow 0, \beta_{13} \rightarrow 0\right.\right.\right.$, $\left.\left.\left.\beta_{23} \rightarrow 0, \beta_{14} \rightarrow 0, \beta_{24} \rightarrow 0, \beta_{25} \rightarrow 0, \beta_{16} \rightarrow 0\right\},\left\{\alpha_{22}, 0,0\right\}\right] / .\left\{\alpha_{22} \rightarrow 0\right\}\right]$

0

$\mathrm{DB} 13=\operatorname{Simplify}\left[\operatorname{Series}\left[D\left[\mathrm{H} 2, \alpha_{13}\right] / .\left\{\alpha_{11} \rightarrow 0, \alpha_{13} \rightarrow 0, \alpha_{24} \rightarrow 0, \beta_{11} \rightarrow 0, \beta_{21} \rightarrow 0, \beta_{12} \rightarrow 0, \beta_{22} \rightarrow 0, \beta_{13} \rightarrow 0\right.\right.\right.$, $\left.\left.\left.\beta_{23} \rightarrow 0, \beta_{14} \rightarrow 0, \beta_{24} \rightarrow 0, \beta_{25} \rightarrow 0, \beta_{16} \rightarrow 0\right\},\left\{\alpha_{22}, 0,0\right\}\right] / .\left\{\alpha_{22} \rightarrow 0\right\}\right]$

0

$\mathrm{DB} 23=$ Simplify $\left[\operatorname{Series}\left[D\left[\mathrm{H} 2, \alpha_{23}\right] / .\left\{\alpha_{11} \rightarrow 0, \alpha_{13} \rightarrow 0, \alpha_{24} \rightarrow 0, \beta_{11} \rightarrow 0, \beta_{21} \rightarrow 0, \beta_{12} \rightarrow 0, \beta_{22} \rightarrow 0, \beta_{13} \rightarrow 0\right.\right.\right.$, $\left.\left.\left.\beta_{23} \rightarrow 0, \beta_{14} \rightarrow 0, \beta_{24} \rightarrow 0, \beta_{25} \rightarrow 0, \beta_{16} \rightarrow 0\right\},\left\{\alpha_{22}, 0,0\right\}\right] / .\left\{\alpha_{22} \rightarrow 0\right\}\right]$

0

$\mathrm{DB} 14=$ Simplify $\left[\right.$ Series $\left[D\left[\mathrm{H} 2, \alpha_{14}\right] / .\left\{\alpha_{11} \rightarrow 0, \alpha_{13} \rightarrow 0, \alpha_{24} \rightarrow 0, \beta_{11} \rightarrow 0, \beta_{21} \rightarrow 0, \beta_{12} \rightarrow 0, \beta_{22} \rightarrow 0, \beta_{13} \rightarrow 0\right.\right.$, $\left.\left.\left.\beta_{23} \rightarrow 0, \beta_{14} \rightarrow 0, \beta_{24} \rightarrow 0, \beta_{25} \rightarrow 0, \beta_{16} \rightarrow 0\right\},\left\{\alpha_{22}, 0,0\right\}\right] / .\left\{\alpha_{22} \rightarrow 0\right\}\right]$

0

$\mathrm{DB} 24=\operatorname{Simplify}\left[\operatorname{Series}\left[D\left[\mathrm{H} 2, \alpha_{24}\right] / .\left\{\alpha_{11} \rightarrow 0, \alpha_{13} \rightarrow 0, \alpha_{24} \rightarrow 0, \beta_{11} \rightarrow 0, \beta_{21} \rightarrow 0, \beta_{12} \rightarrow 0, \beta_{22} \rightarrow 0, \beta_{13} \rightarrow 0\right.\right.\right.$, $\left.\left.\left.\beta_{23} \rightarrow 0, \beta_{14} \rightarrow 0, \beta_{24} \rightarrow 0, \beta_{25} \rightarrow 0, \beta_{16} \rightarrow 0\right\},\left\{\alpha_{22}, 0,0\right\}\right] / .\left\{\alpha_{22} \rightarrow 0\right\}\right]$

0

$\mathrm{DB} 25=$ Simplify $\left[\operatorname{Series}\left[D\left[\mathrm{H} 2, \alpha_{25}\right] / .\left\{\alpha_{11} \rightarrow 0, \alpha_{13} \rightarrow 0, \alpha_{24} \rightarrow 0, \beta_{11} \rightarrow 0, \beta_{21} \rightarrow 0, \beta_{12} \rightarrow 0, \beta_{22} \rightarrow 0, \beta_{13} \rightarrow 0\right.\right.\right.$, $\left.\left.\left.\beta_{23} \rightarrow 0, \beta_{14} \rightarrow 0, \beta_{24} \rightarrow 0, \beta_{25} \rightarrow 0, \beta_{16} \rightarrow 0\right\},\left\{\alpha_{22}, 0,0\right\}\right] / .\left\{\alpha_{22} \rightarrow 0\right\}\right]$

0

$\mathrm{DB} 16=$ Simplify $\left[\operatorname{Series}\left[D\left[\mathrm{H} 2, \alpha_{16}\right] / .\left\{\alpha_{11} \rightarrow 0, \alpha_{13} \rightarrow 0, \alpha_{24} \rightarrow 0, \beta_{11} \rightarrow 0, \beta_{21} \rightarrow 0, \beta_{12} \rightarrow 0, \beta_{22} \rightarrow 0, \beta_{13} \rightarrow 0\right.\right.\right.$, $\left.\left.\left.\beta_{23} \rightarrow 0, \beta_{14} \rightarrow 0, \beta_{24} \rightarrow 0, \beta_{25} \rightarrow 0, \beta_{16} \rightarrow 0\right\},\left\{\alpha_{22}, 0,0\right\}\right] / .\left\{\alpha_{22} \rightarrow 0\right\}\right]$ 
(* Next we find the (right parts of the) reduced equations (from the full Hamiltonian) for the remainder of the variables, calculate the reduced Hamiltonian and check that the equations of the reduced system are the same as the equations from the reduced Hamiltonian *)

DA12 = Simplify[Series $\left[D\left[\mathrm{H} 2, \beta_{12}\right] / \cdot\left\{\alpha_{11} \rightarrow 0, \alpha_{13} \rightarrow 0, \alpha_{24} \rightarrow 0, \beta_{11} \rightarrow 0, \beta_{21} \rightarrow 0, \beta_{12} \rightarrow 0, \beta_{22} \rightarrow 0, \beta_{13} \rightarrow 0\right.\right.$, $\left.\left.\left.\beta_{23} \rightarrow 0, \beta_{14} \rightarrow 0, \beta_{24} \rightarrow 0, \beta_{25} \rightarrow 0, \beta_{16} \rightarrow 0\right\},\left\{\alpha_{22}, 0,0\right\}\right] / .\left\{\alpha_{22} \rightarrow 0\right\}\right]$

$$
\begin{aligned}
& \frac{1}{\left(a_{2}-a_{4}\right)^{3}\left(a_{2}-a_{5}\right)^{3}\left(a_{4}-a_{5}\right)^{2}} 2 \alpha_{12}\left(-a_{2}\left(-a_{5}^{3}\left(b_{2}+b_{4}\right)+a_{4}^{3}\left(b_{2}+b_{5}\right)+a_{2}^{2}\left(-a_{5}\left(b_{2}+b_{4}\right)+a_{4}\left(b_{2}+\right.\right.\right.\right. \\
& \left.\left.\left.b_{5}\right)\right)-2 a_{2}\left(-a_{5}^{2}\left(b_{2}+b_{4}\right)+a_{4}^{2}\left(b_{2}+b_{5}\right)\right)\right)\left(a_{5}\left(\kappa_{2}-\kappa_{4}\right)+a_{2}\left(\kappa_{4}-\kappa_{5}\right)+a_{4}\left(-\kappa_{2}+\kappa_{5}\right)\right)+\left(a _ { 5 } ^ { 3 } \left(b_{2}+\right.\right. \\
& \left.b_{4}\right)+a_{2}^{3}\left(b_{4}-b_{5}\right)-a_{4}^{3}\left(b_{2}+b_{5}\right)+a_{2}^{2}\left(-a_{5}\left(b_{2}+b_{4}\right)+a_{4}\left(b_{2}+b_{5}\right)\right)+a_{2}\left(-a_{5}^{2}\left(b_{2}+b_{4}\right)+a_{4}^{2}\left(b_{2}+\right.\right. \\
& \left.\left.\left.b_{5}\right)\right)\right)\left(a_{5}^{2}\left(\kappa_{2}-\kappa_{4}\right)+a_{2}^{2}\left(\kappa_{4}-\kappa_{5}\right)+a_{4}^{2}\left(-\kappa_{2}+\kappa_{5}\right)\right)-\left(a_{5}^{2}\left(b_{2}+b_{4}\right)+a_{2}^{2}\left(b_{4}-b_{5}\right)-a_{4}^{2}\left(b_{2}+b_{5}\right)+\right. \\
& \left.\left.2 a_{2}\left(-a_{5}\left(b_{2}+b_{4}\right)+a_{4}\left(b_{2}+b_{5}\right)\right)\right)\left(a_{2} a_{5}\left(-a_{2}+a_{5}\right) \kappa_{4}+a_{4}^{2}\left(a_{5} \kappa_{2}-a_{2} \kappa_{5}\right)+a_{4}\left(-a_{5}^{2} \kappa_{2}+a_{2}^{2} \kappa_{5}\right)\right)\right)
\end{aligned}
$$

DA14 $=$ Simplify $\left[\operatorname{Series}\left[D\left[\mathrm{H} 2, \beta_{14}\right] / .\left\{\alpha_{11} \rightarrow 0, \alpha_{13} \rightarrow 0, \alpha_{24} \rightarrow 0, \beta_{11} \rightarrow 0, \beta_{21} \rightarrow 0, \beta_{12} \rightarrow 0, \beta_{22} \rightarrow 0, \beta_{13} \rightarrow 0\right.\right.\right.$, $\left.\left.\left.\beta_{23} \rightarrow 0, \beta_{14} \rightarrow 0, \beta_{24} \rightarrow 0, \beta_{25} \rightarrow 0, \beta_{16} \rightarrow 0\right\},\left\{\alpha_{22}, 0,0\right\}\right] / .\left\{\alpha_{22} \rightarrow 0\right\}\right]$

$$
\begin{aligned}
& \frac{1}{\left(a_{2}-a_{4}\right)^{3}\left(a_{2}-a_{5}\right)^{2}\left(a_{4}-a_{5}\right)^{3}} 2 \alpha_{14}\left(-a_{4}\left(a_{5}^{3}\left(b_{2}+b_{4}\right)-a_{2}^{3}\left(b_{4}+b_{5}\right)+a_{4}^{2}\left(a_{5}\left(b_{2}+b_{4}\right)-a_{2}\left(b_{4}+b_{5}\right)\right)-\right.\right. \\
& \left.2 a_{4}\left(a_{5}^{2}\left(b_{2}+b_{4}\right)-a_{2}^{2}\left(b_{4}+b_{5}\right)\right)\right)\left(a_{5}\left(-\kappa_{2}+\kappa_{4}\right)+a_{4}\left(\kappa_{2}-\kappa_{5}\right)+a_{2}\left(-\kappa_{4}+\kappa_{5}\right)\right)-\left(a _ { 5 } ^ { 3 } \left(b_{2}+\right.\right. \\
& \left.b_{4}\right)+a_{4}^{3}\left(b_{2}-b_{5}\right)-a_{2}^{3}\left(b_{4}+b_{5}\right)+a_{4}^{2}\left(-a_{5}\left(b_{2}+b_{4}\right)+a_{2}\left(b_{4}+b_{5}\right)\right)+a_{4}\left(-a_{5}^{2}\left(b_{2}+b_{4}\right)+a_{2}^{2}\left(b_{4}+\right.\right. \\
& \left.\left.\left.b_{5}\right)\right)\right)\left(a_{5}^{2}\left(-\kappa_{2}+\kappa_{4}\right)+a_{4}^{2}\left(\kappa_{2}-\kappa_{5}\right)+a_{2}^{2}\left(-\kappa_{4}+\kappa_{5}\right)\right)+\left(-a_{5}^{2}\left(b_{2}+b_{4}\right)+a_{4}^{2}\left(-b_{2}+b_{5}\right)+a_{2}^{2}\left(b_{4}+b_{5}\right)+\right. \\
& \left.\left.2 a_{4}\left(a_{5}\left(b_{2}+b_{4}\right)-a_{2}\left(b_{4}+b_{5}\right)\right)\right)\left(a_{2} a_{5}\left(-a_{2}+a_{5}\right) \kappa_{4}+a_{4}^{2}\left(a_{5} \kappa_{2}-a_{2} \kappa_{5}\right)+a_{4}\left(-a_{5}^{2} \kappa_{2}+a_{2}^{2} \kappa_{5}\right)\right)\right) \\
& \text { Da1 }=\text { Simplify }\left[\text { Series } \left[D\left[\mathrm{H} 2, \kappa_{1}\right] / .\left\{\alpha_{11} \rightarrow 0, \alpha_{13} \rightarrow 0, \alpha_{24} \rightarrow 0, \beta_{21} \rightarrow 0, \beta_{12} \rightarrow 0, \beta_{23} \rightarrow 0, \beta_{14} \rightarrow 0\right. \text {, }\right.\right. \\
& \left.\left.\left.\beta_{25} \rightarrow 0, \beta_{16} \rightarrow 0\right\},\left\{\alpha_{22}, 0,0\right\}\right] / \cdot\left\{\alpha_{22} \rightarrow 0\right\}\right] \\
& \frac{1}{\left(a_{1}-a_{3}\right)^{2}\left(a_{1}-a_{6}\right)^{2}\left(a_{3}-a_{6}\right)} 2\left(a_{3}^{3}\left(\kappa_{1}-\kappa_{6}\right)+a_{3}^{2}\left(a_{6}\left(3 \kappa_{1}-2 \kappa_{6}\right)-a_{1} \kappa_{6}\right)+a_{6}\left(a_{1} a_{6} \kappa_{3}+a_{6}^{2}\left(-\kappa_{1}+\right.\right.\right. \\
& \left.\left.\left.\kappa_{3}\right)+a_{1}^{2}\left(-2 \kappa_{3}+\kappa_{6}\right)\right)+a_{3}\left(a_{6}^{2}\left(-3 \kappa_{1}+2 \kappa_{3}\right)-a_{1}^{2}\left(\kappa_{3}-2 \kappa_{6}\right)+a_{1} a_{6}\left(-\kappa_{3}+\kappa_{6}\right)\right)\right) \\
& \mathrm{Da} 2=\text { Simplify[Series }\left[D\left[\mathrm{H} 2, \kappa_{2}\right] / .\left\{\alpha_{11} \rightarrow 0, \alpha_{13} \rightarrow 0, \alpha_{24} \rightarrow 0, \beta_{11} \rightarrow 0, \beta_{21} \rightarrow 0, \beta_{12} \rightarrow 0, \beta_{22} \rightarrow 0\right. \text {, }\right. \\
& \left.\left.\left.\beta_{13} \rightarrow 0, \beta_{23} \rightarrow 0, \beta_{14} \rightarrow 0, \beta_{24} \rightarrow 0, \beta_{25} \rightarrow 0, \beta_{16} \rightarrow 0\right\},\left\{\alpha_{22}, 0,0\right\}\right] / .\left\{\alpha_{22} \rightarrow 0\right\}\right] \\
& \frac{1}{\left(a_{2}-a_{4}\right)^{2}\left(a_{2}-a_{5}\right)^{2}\left(a_{4}-a_{5}\right)} 2\left(a_{4}^{3}\left(\kappa_{2}-\kappa_{5}\right)+a_{4}^{2}\left(a_{5}\left(3 \kappa_{2}-2 \kappa_{5}\right)-a_{2} \kappa_{5}\right)+a_{5}\left(a_{2} a_{5} \kappa_{4}+a_{5}^{2}\left(-\kappa_{2}+\right.\right.\right. \\
& \left.\left.\left.\kappa_{4}\right)+a_{2}^{2}\left(-2 \kappa_{4}+\kappa_{5}\right)\right)+a_{4}\left(a_{5}^{2}\left(-3 \kappa_{2}+2 \kappa_{4}\right)-a_{2}^{2}\left(\kappa_{4}-2 \kappa_{5}\right)+a_{2} a_{5}\left(-\kappa_{4}+\kappa_{5}\right)\right)\right) \\
& \text { Da3 = Simplify[Series }\left[D\left[\mathrm{H} 2, \kappa_{3}\right] / .\left\{\alpha_{11} \rightarrow 0, \alpha_{13} \rightarrow 0, \alpha_{24} \rightarrow 0, \beta_{11} \rightarrow 0, \beta_{21} \rightarrow 0, \beta_{12} \rightarrow 0, \beta_{22} \rightarrow 0\right. \text {, }\right. \\
& \left.\left.\left.\beta_{13} \rightarrow 0, \beta_{23} \rightarrow 0, \beta_{14} \rightarrow 0, \beta_{24} \rightarrow 0, \beta_{25} \rightarrow 0, \beta_{16} \rightarrow 0\right\},\left\{\alpha_{22}, 0,0\right\}\right] / .\left\{\alpha_{22} \rightarrow 0\right\}\right](* *)
\end{aligned}
$$




$$
\begin{aligned}
& \frac{1}{\left(a_{1}-a_{3}\right)^{2}\left(a_{1}-a_{6}\right)\left(a_{3}-a_{6}\right)^{2}} 2\left(a_{1}^{3}\left(\kappa_{3}-\kappa_{6}\right)+a_{1}^{2}\left(a_{6}\left(3 \kappa_{3}-2 \kappa_{6}\right)-a_{3} \kappa_{6}\right)+a_{6}\left(a_{3} a_{6} \kappa_{1}+a_{6}^{2}\left(\kappa_{1}-\right.\right.\right. \\
& \left.\left.\left.\kappa_{3}\right)+a_{3}^{2}\left(-2 \kappa_{1}+\kappa_{6}\right)\right)+a_{1}\left(a_{6}^{2}\left(2 \kappa_{1}-3 \kappa_{3}\right)-a_{3}^{2}\left(\kappa_{1}-2 \kappa_{6}\right)+a_{3} a_{6}\left(-\kappa_{1}+\kappa_{6}\right)\right)\right) \\
& \mathrm{Da} 4=\text { Simplify }\left[\text { Series } \left[D\left[\mathrm{H} 2, \kappa_{4}\right] / .\left\{\alpha_{11} \rightarrow 0, \alpha_{13} \rightarrow 0, \alpha_{24} \rightarrow 0, \beta_{11} \rightarrow 0, \beta_{21} \rightarrow 0, \beta_{12} \rightarrow 0, \beta_{22} \rightarrow 0\right. \text {, }\right.\right. \\
& \left.\left.\left.\beta_{13} \rightarrow 0, \beta_{23} \rightarrow 0, \beta_{14} \rightarrow 0, \beta_{24} \rightarrow 0, \beta_{25} \rightarrow 0, \beta_{16} \rightarrow 0\right\},\left\{\alpha_{22}, 0,0\right\}\right] / .\left\{\alpha_{22} \rightarrow 0\right\}\right] \\
& \frac{1}{\left(a_{2}-a_{4}\right)^{2}\left(a_{2}-a_{5}\right)\left(a_{4}-a_{5}\right)^{2}} 2\left(a_{2}^{3}\left(\kappa_{4}-\kappa_{5}\right)+a_{2}^{2}\left(a_{5}\left(3 \kappa_{4}-2 \kappa_{5}\right)-a_{4} \kappa_{5}\right)+a_{5}\left(a_{4} a_{5} \kappa_{2}+a_{5}^{2}\left(\kappa_{2}-\right.\right.\right. \\
& \left.\left.\left.\kappa_{4}\right)+a_{4}^{2}\left(-2 \kappa_{2}+\kappa_{5}\right)\right)+a_{2}\left(a_{5}^{2}\left(2 \kappa_{2}-3 \kappa_{4}\right)-a_{4}^{2}\left(\kappa_{2}-2 \kappa_{5}\right)+a_{4} a_{5}\left(-\kappa_{2}+\kappa_{5}\right)\right)\right) \\
& \text { Da5 }=\text { Simplify }\left[\operatorname { S e r i e s } \left[D\left[\mathrm{H} 2, \kappa_{5}\right] / .\left\{\alpha_{11} \rightarrow 0, \alpha_{13} \rightarrow 0, \alpha_{24} \rightarrow 0, \beta_{11} \rightarrow 0, \beta_{21} \rightarrow 0, \beta_{12} \rightarrow 0, \beta_{22} \rightarrow 0\right. \text {, }\right.\right. \\
& \left.\left.\left.\beta_{13} \rightarrow 0, \beta_{23} \rightarrow 0, \beta_{14} \rightarrow 0, \beta_{24} \rightarrow 0, \beta_{25} \rightarrow 0, \beta_{16} \rightarrow 0\right\},\left\{\alpha_{22}, 0,0\right\}\right] / .\left\{\alpha_{22} \rightarrow 0\right\}\right] \\
& -\frac{1}{\left(a_{2}-a_{4}\right)\left(a_{2}-a_{5}\right)^{2}\left(a_{4}-a_{5}\right)^{2}} 2\left(a_{2}^{2}\left(a_{5} \kappa_{4}+a_{4}\left(2 \kappa_{4}-3 \kappa_{5}\right)\right)-a_{4}\left(a_{4} a_{5} \kappa_{2}+a_{5}^{2}\left(-2 \kappa_{2}+\kappa_{4}\right)+a_{4}^{2}\left(\kappa_{2}-\right.\right.\right. \\
& \left.\left.\left.\kappa_{5}\right)\right)+a_{2}^{3}\left(\kappa_{4}-\kappa_{5}\right)+a_{2}\left(a_{5}^{2}\left(\kappa_{2}-2 \kappa_{4}\right)+a_{4} a_{5}\left(\kappa_{2}-\kappa_{4}\right)+a_{4}^{2}\left(-2 \kappa_{2}+3 \kappa_{5}\right)\right)\right) \\
& \text { Da6 }=\text { Simplify }\left[\text { Series } \left[D\left[\mathrm{H} 2, \kappa_{6}\right] / .\left\{\alpha_{11} \rightarrow 0, \alpha_{13} \rightarrow 0, \alpha_{24} \rightarrow 0, \beta_{11} \rightarrow 0, \beta_{21} \rightarrow 0, \beta_{12} \rightarrow 0, \beta_{22} \rightarrow 0\right. \text {, }\right.\right. \\
& \left.\left.\left.\beta_{13} \rightarrow 0, \beta_{23} \rightarrow 0, \beta_{14} \rightarrow 0, \beta_{24} \rightarrow 0, \beta_{25} \rightarrow 0, \beta_{16} \rightarrow 0\right\},\left\{\alpha_{22}, 0,0\right\}\right] / .\left\{\alpha_{22} \rightarrow 0\right\}\right] \\
& -\frac{1}{\left(a_{1}-a_{3}\right)\left(a_{1}-a_{6}\right)^{2}\left(a_{3}-a_{6}\right)^{2}} 2\left(a_{1}^{2}\left(a_{6} \kappa_{3}+a_{3}\left(2 \kappa_{3}-3 \kappa_{6}\right)\right)-a_{3}\left(a_{3} a_{6} \kappa_{1}+a_{6}^{2}\left(-2 \kappa_{1}+\kappa_{3}\right)+a_{3}^{2}\left(\kappa_{1}-\right.\right.\right. \\
& \left.\left.\left.\kappa_{6}\right)\right)+a_{1}^{3}\left(\kappa_{3}-\kappa_{6}\right)+a_{1}\left(a_{6}^{2}\left(\kappa_{1}-2 \kappa_{3}\right)+a_{3} a_{6}\left(\kappa_{1}-\kappa_{3}\right)+a_{3}^{2}\left(-2 \kappa_{1}+3 \kappa_{6}\right)\right)\right)
\end{aligned}
$$

\section{Simplify[Da1 + Da3 + Da6 $]$}

$\frac{2\left(a_{6}\left(-\kappa_{1}+\kappa_{3}\right)+a_{3}\left(\kappa_{1}-\kappa_{6}\right)+a_{1}\left(-\kappa_{3}+\kappa_{6}\right)\right)}{\left(a_{1}-a_{3}\right)\left(a_{1}-a_{6}\right)\left(a_{3}-a_{6}\right)}$

$\left(a_{1}-a_{3}\right)\left(a_{1}-a_{6}\right)\left(a_{3}-a_{6}\right)$

(*The dependence of $a_{s}$ on $b_{s}$ has been switched on at calculating the D $\kappa_{s}$. It does not affect the result : *)

$\mathrm{D} \kappa 1=\operatorname{Simplify}\left[\right.$ Series$\left[-D\left[\mathrm{H} 2 / .\left\{b_{1} \rightarrow f\left[a_{1}\right]\right\}, a_{1}\right] / .\left\{\alpha_{11} \rightarrow 0, \alpha_{13} \rightarrow 0, \alpha_{24} \rightarrow 0, \beta_{11} \rightarrow 0, \beta_{21} \rightarrow 0\right.\right.$, $\left.\left.\left.\beta_{12} \rightarrow 0, \beta_{22} \rightarrow 0, \beta_{13} \rightarrow 0, \beta_{23} \rightarrow 0, \beta_{14} \rightarrow 0, \beta_{24} \rightarrow 0, \beta_{25} \rightarrow 0, \beta_{16} \rightarrow 0\right\},\left\{\alpha_{22}, 0,0\right\}\right] / .\left\{\alpha_{22} \rightarrow 0\right\}\right]$

$$
\begin{aligned}
& \frac{1}{\left(a_{1}-a_{3}\right)^{3}\left(a_{1}-a_{6}\right)^{3}\left(a_{3}-a_{6}\right)^{2}} 2\left(-a_{6}^{5}\left(\kappa_{1}-\kappa_{3}\right)^{2}+a_{3} a_{6}^{4}\left(-3 \kappa_{1}^{2}+5 \kappa_{1} \kappa_{3}-2 \kappa_{3}^{2}\right)+a_{3}^{2} a_{6}^{3}\left(\kappa_{1}-\kappa_{3}\right)\left(4 \kappa_{1}-\right.\right. \\
& \left.3 \kappa_{6}\right)+a_{3}^{3} a_{6}^{2}\left(4 \kappa_{1}-3 \kappa_{3}\right)\left(\kappa_{1}-\kappa_{6}\right)-a_{3}^{5}\left(\kappa_{1}-\kappa_{6}\right)^{2}+a_{1}^{4}\left(a_{3}\left(\kappa_{3}-2 \kappa_{6}\right)+a_{6}\left(2 \kappa_{3}-\kappa_{6}\right)\right)\left(\kappa_{3}-\kappa_{6}\right)- \\
& 3 a_{1}^{2}\left(a_{3}-a_{6}\right)\left(a_{6}\left(-\kappa_{1}+\kappa_{3}\right)+a_{3}\left(\kappa_{1}-\kappa_{6}\right)\right)\left(a_{6} \kappa_{3}+a_{3} \kappa_{6}\right)+a_{3}^{4} a_{6}\left(-3 \kappa_{1}^{2}+5 \kappa_{1} \kappa_{6}-2 \kappa_{6}{ }^{2}\right)+ \\
& a_{1}\left(a_{6}^{4}\left(2 \kappa_{1}^{2}-3 \kappa_{1} \kappa_{3}+\kappa_{3}^{2}\right)+a_{3}^{3} a_{6}\left(4 \kappa_{1}+3 \kappa_{3}-5 \kappa_{6}\right)\left(\kappa_{1}-\kappa_{6}\right)+a_{3} a_{6}^{3}\left(\kappa_{1}-\kappa_{3}\right)\left(4 \kappa_{1}-5 \kappa_{3}+3 \kappa_{6}\right)+\right. \\
& \left.a_{3}^{4}\left(2 \kappa_{1}^{2}-3 \kappa_{1} \kappa_{6}+\kappa_{6}{ }^{2}\right)+3 a_{3}^{2} a_{6}^{2}\left(-4 \kappa_{1}^{2}-2 \kappa_{3} \kappa_{6}+3 \kappa_{1}\left(\kappa_{3}+\kappa_{6}\right)\right)\right)-a_{1}^{3}\left(a _ { 3 } a _ { 6 } \left(\kappa_{3}^{2}-6 \kappa_{3} \kappa_{6}+\kappa_{6}{ }^{2}+\right.\right. \\
& \left.\left.\left.2 \kappa_{1}\left(\kappa_{3}+\kappa_{6}\right)\right)+a_{6}^{2}\left(\kappa_{3}\left(5 \kappa_{3}-3 \kappa_{6}\right)+\kappa_{1}\left(-4 \kappa_{3}+2 \kappa_{6}\right)\right)+a_{3}^{2}\left(2 \kappa_{1}\left(\kappa_{3}-2 \kappa_{6}\right)+\kappa_{6}\left(-3 \kappa_{3}+5 \kappa_{6}\right)\right)\right)\right)
\end{aligned}
$$


$\mathrm{D} \kappa 2=\operatorname{Simplify}\left[\operatorname{Series}\left[-D\left[\mathrm{H} 2 / .\left\{b_{2} \rightarrow f\left[a_{2}\right]\right\}, a_{2}\right] / .\left\{\alpha_{11} \rightarrow 0, \alpha_{13} \rightarrow 0, \alpha_{24} \rightarrow 0, \beta_{11} \rightarrow 0, \beta_{21} \rightarrow 0\right.\right.\right.$, $\left.\left.\left.\beta_{12} \rightarrow 0, \beta_{22} \rightarrow 0, \beta_{13} \rightarrow 0, \beta_{23} \rightarrow 0, \beta_{14} \rightarrow 0, \beta_{24} \rightarrow 0, \beta_{25} \rightarrow 0, \beta_{16} \rightarrow 0\right\},\left\{\alpha_{22}, 0,0\right\}\right] / .\left\{\alpha_{22} \rightarrow 0\right\}\right]$ $\frac{1}{\left(a_{2}-a_{4}\right)^{3}\left(a_{2}-a_{5}\right)^{3}\left(a_{4}-a_{5}\right)^{2}} 2\left(-a_{5}^{5}\left(\kappa_{2}-\kappa_{4}\right)^{2}+a_{4} a_{5}^{4}\left(-3 \kappa_{2}^{2}+5 \kappa_{2} \kappa_{4}-2 \kappa_{4}^{2}\right)+a_{4}^{2} a_{5}^{3}\left(\kappa_{2}-\kappa_{4}\right)\left(4 \kappa_{2}-\right.\right.$ $\left.3 \kappa_{5}\right)+a_{4}^{3} a_{5}^{2}\left(4 \kappa_{2}-3 \kappa_{4}\right)\left(\kappa_{2}-\kappa_{5}\right)-a_{4}^{5}\left(\kappa_{2}-\kappa_{5}\right)^{2}+a_{2}^{4}\left(a_{4}\left(\kappa_{4}-2 \kappa_{5}\right)+a_{5}\left(2 \kappa_{4}-\kappa_{5}\right)\right)\left(\kappa_{4}-\kappa_{5}\right)-$ $3 a_{2}^{2}\left(a_{4}-a_{5}\right)\left(a_{5}\left(-\kappa_{2}+\kappa_{4}\right)+a_{4}\left(\kappa_{2}-\kappa_{5}\right)\right)\left(a_{5} \kappa_{4}+a_{4} \kappa_{5}\right)+a_{4}^{4} a_{5}\left(-3 \kappa_{2}^{2}+5 \kappa_{2} \kappa_{5}-2 \kappa_{5}^{2}\right)+$ $a_{2}\left(a_{5}^{4}\left(2 \kappa_{2}^{2}-3 \kappa_{2} \kappa_{4}+\kappa_{4}^{2}\right)+a_{4}^{3} a_{5}\left(4 \kappa_{2}+3 \kappa_{4}-5 \kappa_{5}\right)\left(\kappa_{2}-\kappa_{5}\right)+a_{4} a_{5}^{3}\left(\kappa_{2}-\kappa_{4}\right)\left(4 \kappa_{2}-5 \kappa_{4}+3 \kappa_{5}\right)+\right.$ $\left.a_{4}^{4}\left(2 \kappa_{2}^{2}-3 \kappa_{2} \kappa_{5}+\kappa_{5}^{2}\right)+3 a_{4}^{2} a_{5}^{2}\left(-4 \kappa_{2}^{2}-2 \kappa_{4} \kappa_{5}+3 \kappa_{2}\left(\kappa_{4}+\kappa_{5}\right)\right)\right)-a_{2}^{3}\left(a_{4} a_{5}\left(\kappa_{4}^{2}-6 \kappa_{4} \kappa_{5}+\kappa_{5}^{2}+\right.\right.$ $\left.\left.\left.2 \kappa_{2}\left(\kappa_{4}+\kappa_{5}\right)\right)+a_{5}^{2}\left(\kappa_{4}\left(5 \kappa_{4}-3 \kappa_{5}\right)+\kappa_{2}\left(-4 \kappa_{4}+2 \kappa_{5}\right)\right)+a_{4}^{2}\left(2 \kappa_{2}\left(\kappa_{4}-2 \kappa_{5}\right)+\kappa_{5}\left(-3 \kappa_{4}+5 \kappa_{5}\right)\right)\right)\right)$

$\mathrm{D} \kappa 3=$ Simplify[Series $\left[-D\left[\mathrm{H} 2 / .\left\{b_{3} \rightarrow f\left[a_{3}\right]\right\}, a_{3}\right] / .\left\{\alpha_{11} \rightarrow 0, \alpha_{13} \rightarrow 0, \alpha_{24} \rightarrow 0, \beta_{11} \rightarrow 0, \beta_{21} \rightarrow 0\right.\right.$, $\left.\left.\left.\beta_{12} \rightarrow 0, \beta_{22} \rightarrow 0, \beta_{13} \rightarrow 0, \beta_{23} \rightarrow 0, \beta_{14} \rightarrow 0, \beta_{24} \rightarrow 0, \beta_{25} \rightarrow 0, \beta_{16} \rightarrow 0\right\},\left\{\alpha_{22}, 0,0\right\}\right] / .\left\{\alpha_{22} \rightarrow 0\right\}\right]$ $\frac{1}{\left(a_{1}-a_{3}\right)^{3}\left(a_{1}-a_{6}\right)^{2}\left(a_{3}-a_{6}\right)^{3}} 2\left(a_{6}^{2}\left(\kappa_{1}-\kappa_{3}\right)-2 a_{3}\left(a_{6}\left(\kappa_{1}-\kappa_{3}\right)+a_{1}\left(\kappa_{3}-\kappa_{6}\right)\right)+a_{3}^{2}\left(\kappa_{1}-\kappa_{6}\right)+\right.$ $\left.a_{1}^{2}\left(\kappa_{3}-\kappa_{6}\right)\right)\left(a_{1}^{3}\left(\kappa_{3}-\kappa_{6}\right)+a_{1}^{2}\left(a_{6}\left(3 \kappa_{3}-2 \kappa_{6}\right)-a_{3} \kappa_{6}\right)+a_{6}\left(a_{3} a_{6} \kappa_{1}+a_{6}^{2}\left(\kappa_{1}-\kappa_{3}\right)+a_{3}^{2}\left(-2 \kappa_{1}+\right.\right.\right.$ $\left.\left.\left.\kappa_{6}\right)\right)+a_{1}\left(a_{6}^{2}\left(2 \kappa_{1}-3 \kappa_{3}\right)-a_{3}^{2}\left(\kappa_{1}-2 \kappa_{6}\right)+a_{3} a_{6}\left(-\kappa_{1}+\kappa_{6}\right)\right)\right)$

(* The obtained expression for $\mathrm{D} \kappa 3$ is simpler than for other $\kappa$ 's. This enabled us to simplify the others and finally to conjecture the relations between the derivatives of $\kappa$ and $a *$ )

$\mathrm{D} \kappa 4=$ Simplify[Series[-D[H2/. $\left.\left\{b_{4} \rightarrow f\left[a_{4}\right]\right\}, a_{4}\right] / .\left\{\alpha_{11} \rightarrow 0, \alpha_{13} \rightarrow 0, \alpha_{24} \rightarrow 0, \beta_{11} \rightarrow 0, \beta_{21} \rightarrow 0\right.$, $\left.\left.\left.\beta_{12} \rightarrow 0, \beta_{22} \rightarrow 0, \beta_{13} \rightarrow 0, \beta_{23} \rightarrow 0, \beta_{14} \rightarrow 0, \beta_{24} \rightarrow 0, \beta_{25} \rightarrow 0, \beta_{16} \rightarrow 0\right\},\left\{\alpha_{22}, 0,0\right\}\right] / .\{\alpha 22 \rightarrow 0\}\right]$ $\frac{1}{\left(a_{2}-a_{4}\right)^{3}\left(a_{2}-a_{5}\right)^{2}\left(a_{4}-a_{5}\right)^{3}} 2\left(a_{2}^{5}\left(\kappa_{4}-\kappa_{5}\right)^{2}-a_{2}^{4}\left(\kappa_{4}-\kappa_{5}\right)\left(a_{4}\left(2 \kappa_{4}-\kappa_{5}\right)+a_{5}\left(-3 \kappa_{4}+2 \kappa_{5}\right)\right)+\right.$
$a_{2}^{3}\left(\kappa_{4}-\kappa_{5}\right)\left(a_{5}^{2}\left(3 \kappa_{2}-4 \kappa_{4}\right)+3 a_{4}^{2} \kappa_{5}+a_{4} a_{5}\left(-3 \kappa_{2}-4 \kappa_{4}+5 \kappa_{5}\right)\right)+a_{2}\left(a_{5}^{4}\left(2 \kappa_{2}^{2}-5 \kappa_{2} \kappa_{4}+3 \kappa_{4}^{2}\right)-\right.$
$a_{4} a_{5}^{3}\left(\kappa_{2}-\kappa_{4}\right)\left(5 \kappa_{2}-4 \kappa_{4}-3 \kappa_{5}\right)+3 a_{4}^{2} a_{5}^{2}\left(\kappa_{2}^{2}-2 \kappa_{2} \kappa_{4}+\kappa_{4} \kappa_{5}\right)-a_{4}^{4}\left(\kappa_{2}^{2}-3 \kappa_{2} \kappa_{5}+2 \kappa_{5}^{2}\right)+$
$\left.a_{4}^{3} a_{5}\left(\kappa_{2}^{2}+2 \kappa_{2}\left(\kappa_{4}-3 \kappa_{5}\right)+\kappa_{5}\left(2 \kappa_{4}+\kappa_{5}\right)\right)\right)+a_{5}\left(a_{5}^{4}\left(\kappa_{2}-\kappa_{4}\right)^{2}+3 a_{4}^{2} a_{5}^{2} \kappa_{2}\left(-\kappa_{2}+\kappa_{4}\right)-a_{4} a_{5}^{3}\left(\kappa_{2}^{2}-\right.\right.$
$\left.\left.3 \kappa_{2} \kappa_{4}+2 \kappa_{4}^{2}\right)-a_{4}^{4}\left(2 \kappa_{2}^{2}-3 \kappa_{2} \kappa_{5}+\kappa_{5}^{2}\right)+a_{4}^{3} a_{5}\left(5 \kappa_{2}^{2}+2 \kappa_{4} \kappa_{5}-\kappa_{2}\left(4 \kappa_{4}+3 \kappa_{5}\right)\right)\right)+a_{2}^{2}\left(a_{5}^{3}\left(\kappa_{2}-\right.\right.$
$\left.\kappa_{4}\right)\left(4 \kappa_{4}-3 \kappa_{5}\right)+3 a_{4}^{2} a_{5}\left(\kappa_{2} \kappa_{4}+\kappa_{5}\left(-2 \kappa_{4}+\kappa_{5}\right)\right)+3 a_{4} a_{5}^{2}\left(\kappa_{4}\left(4 \kappa_{4}-3 \kappa_{5}\right)+\kappa_{2}\left(-3 \kappa_{4}+2 \kappa_{5}\right)\right)+$
$\left.\left.a_{4}^{3}\left(\kappa_{2}\left(2 \kappa_{4}-3 \kappa_{5}\right)+\kappa_{5}\left(-4 \kappa_{4}+5 \kappa_{5}\right)\right)\right)\right)$ $\mathrm{D} \kappa 5=$ Simplify $\left[\right.$ Series $\left[-D\left[\mathrm{H} 2 / .\left\{b_{5} \rightarrow f\left[a_{5}\right]\right\}, a_{5}\right] / .\left\{\alpha_{11} \rightarrow 0, \alpha_{13} \rightarrow 0, \alpha_{24} \rightarrow 0, \beta_{11} \rightarrow 0, \beta_{21} \rightarrow 0\right.\right.$, $\left.\left.\left.\beta_{12} \rightarrow 0, \beta_{22} \rightarrow 0, \beta_{13} \rightarrow 0, \beta_{23} \rightarrow 0, \beta_{14} \rightarrow 0, \beta_{24} \rightarrow 0, \beta_{25} \rightarrow 0, \beta_{16} \rightarrow 0\right\},\left\{\alpha_{22}, 0,0\right\}\right] / .\left\{\alpha_{22} \rightarrow 0\right\}\right]$

$$
\begin{aligned}
& -\frac{1}{\left(a_{2}-a_{4}\right)^{2}\left(a_{2}-a_{5}\right)^{3}\left(a_{4}-a_{5}\right)^{3}} 2\left(-a_{2}^{3}\left(3 a_{5}^{2} \kappa_{4}+a_{4}^{2}\left(3 \kappa_{2}-4 \kappa_{5}\right)+a_{4} a_{5}\left(-3 \kappa_{2}+5 \kappa_{4}-4 \kappa_{5}\right)\right)\left(\kappa_{4}-\right.\right. \\
& \left.\kappa_{5}\right)+a_{2}^{4}\left(a_{4}\left(2 \kappa_{4}-3 \kappa_{5}\right)-a_{5}\left(\kappa_{4}-2 \kappa_{5}\right)\right)\left(\kappa_{4}-\kappa_{5}\right)+a_{2}^{5}\left(\kappa_{4}-\kappa_{5}\right)^{2}+a_{2}\left(-a_{5}^{4}\left(\kappa_{2}^{2}-3 \kappa_{2} \kappa_{4}+2 \kappa_{4}^{2}\right)-\right. \\
& a_{4}^{3} a_{5}\left(5 \kappa_{2}-3 \kappa_{4}-4 \kappa_{5}\right)\left(\kappa_{2}-\kappa_{5}\right)+3 a_{4}^{2} a_{5}^{2}\left(\kappa_{2}^{2}-2 \kappa_{2} \kappa_{5}+\kappa_{4} \kappa_{5}\right)+a_{4}^{4}\left(2 \kappa_{2}^{2}-5 \kappa_{2} \kappa_{5}+3 \kappa_{5}{ }^{2}\right)+
\end{aligned}
$$




$$
\begin{aligned}
& \left.a_{4} a_{5}^{3}\left(\kappa_{2}^{2}+\kappa_{2}\left(-6 \kappa_{4}+2 \kappa_{5}\right)+\kappa_{4}\left(\kappa_{4}+2 \kappa_{5}\right)\right)\right)+a_{2}^{2}\left(a_{4}^{3}\left(3 \kappa_{4}-4 \kappa_{5}\right)\left(-\kappa_{2}+\kappa_{5}\right)+3 a_{4} a_{5}^{2}\left(\kappa_{4}^{2}+\right.\right. \\
& \left.\kappa_{2} \kappa_{5}-2 \kappa_{4} \kappa_{5}\right)+a_{5}^{3}\left(\kappa_{4}\left(5 \kappa_{4}-4 \kappa_{5}\right)+\kappa_{2}\left(-3 \kappa_{4}+2 \kappa_{5}\right)\right)+3 a_{4}^{2} a_{5}\left(\kappa_{2}\left(2 \kappa_{4}-3 \kappa_{5}\right)+\kappa_{5}\left(-3 \kappa_{4}+\right.\right. \\
& \left.\left.\left.4 \kappa_{5}\right)\right)\right)+a_{4}\left(-a_{5}^{4}\left(2 \kappa_{2}^{2}-3 \kappa_{2} \kappa_{4}+\kappa_{4}^{2}\right)+a_{4}^{4}\left(\kappa_{2}-\kappa_{5}\right)^{2}+3 a_{4}^{2} a_{5}^{2} \kappa_{2}\left(-\kappa_{2}+\kappa_{5}\right)-a_{4}^{3} a_{5}\left(\kappa_{2}^{2}-3 \kappa_{2} \kappa_{5}+\right.\right. \\
& \left.\left.\left.2 \kappa_{5}^{2}\right)+a_{4} a_{5}^{3}\left(5 \kappa_{2}^{2}+2 \kappa_{4} \kappa_{5}-\kappa_{2}\left(3 \kappa_{4}+4 \kappa_{5}\right)\right)\right)\right) \\
& \text { D } 66=\text { Simplify }\left[\text { Series } \left[-D\left[\mathrm{H} 2 / .\left\{b_{6} \rightarrow f\left[a_{6}\right]\right\}, a_{6}\right] / .\left\{\alpha_{11} \rightarrow 0, \alpha_{13} \rightarrow 0, \alpha_{24} \rightarrow 0, \beta_{11} \rightarrow 0, \beta_{21} \rightarrow 0\right. \text {, }\right.\right. \\
& \left.\left.\left.\beta_{12} \rightarrow 0, \beta_{22} \rightarrow 0, \beta_{13} \rightarrow 0, \beta_{23} \rightarrow 0, \beta_{14} \rightarrow 0, \beta_{24} \rightarrow 0, \beta_{25} \rightarrow 0, \beta_{16} \rightarrow 0\right\},\left\{\alpha_{22}, 0,0\right\}\right] / .\left\{\alpha_{22} \rightarrow 0\right\}\right] \\
& -\frac{1}{\left(a_{1}-a_{3}\right)^{2}\left(a_{1}-a_{6}\right)^{3}\left(a_{3}-a_{6}\right)^{3}} 2\left(-a_{1}^{3}\left(3 a_{6}^{2} \kappa_{3}+a_{3}^{2}\left(3 \kappa_{1}-4 \kappa_{6}\right)+a_{3} a_{6}\left(-3 \kappa_{1}+5 \kappa_{3}-4 \kappa_{6}\right)\right)\left(\kappa_{3}-\right.\right. \\
& \left.\kappa_{6}\right)+a_{1}^{4}\left(a_{3}\left(2 \kappa_{3}-3 \kappa_{6}\right)-a_{6}\left(\kappa_{3}-2 \kappa_{6}\right)\right)\left(\kappa_{3}-\kappa_{6}\right)+a_{1}^{5}\left(\kappa_{3}-\kappa_{6}\right)^{2}+a_{1}\left(-a_{6}^{4}\left(\kappa_{1}^{2}-3 \kappa_{1} \kappa_{3}+2 \kappa_{3}^{2}\right)-\right. \\
& a_{3}^{3} a_{6}\left(5 \kappa_{1}-3 \kappa_{3}-4 \kappa_{6}\right)\left(\kappa_{1}-\kappa_{6}\right)+3 a_{3}^{2} a_{6}^{2}\left(\kappa_{1}^{2}-2 \kappa_{1} \kappa_{6}+\kappa_{3} \kappa_{6}\right)+a_{3}^{4}\left(2 \kappa_{1}^{2}-5 \kappa_{1} \kappa_{6}+3 \kappa_{6}^{2}\right)+ \\
& \left.a_{3} a_{6}^{3}\left(\kappa_{1}^{2}+\kappa_{1}\left(-6 \kappa_{3}+2 \kappa_{6}\right)+\kappa_{3}\left(\kappa_{3}+2 \kappa_{6}\right)\right)\right)+a_{1}^{2}\left(a_{3}^{3}\left(3 \kappa_{3}-4 \kappa_{6}\right)\left(-\kappa_{1}+\kappa_{6}\right)+3 a_{3} a_{6}^{2}\left(\kappa_{3}^{2}+\right.\right. \\
& \left.\kappa_{1} \kappa_{6}-2 \kappa_{3} \kappa_{6}\right)+a_{6}^{3}\left(\kappa_{3}\left(5 \kappa_{3}-4 \kappa_{6}\right)+\kappa_{1}\left(-3 \kappa_{3}+2 \kappa_{6}\right)\right)+3 a_{3}^{2} a_{6}\left(\kappa_{1}\left(2 \kappa_{3}-3 \kappa_{6}\right)+\kappa_{6}\left(-3 \kappa_{3}+\right.\right. \\
& \left.\left.\left.4 \kappa_{6}\right)\right)\right)+a_{3}\left(-a_{6}^{4}\left(2 \kappa_{1}^{2}-3 \kappa_{1} \kappa_{3}+\kappa_{3}^{2}\right)+a_{3}^{4}\left(\kappa_{1}-\kappa_{6}\right)^{2}+3 a_{3}^{2} a_{6}^{2} \kappa_{1}\left(-\kappa_{1}+\kappa_{6}\right)-a_{3}^{3} a_{6}\left(\kappa_{1}^{2}-3 \kappa_{1} \kappa_{6}+\right.\right. \\
& \left.\left.\left.2 \kappa_{6}{ }^{2}\right)+a_{3} a_{6}^{3}\left(5 \kappa_{1}^{2}+2 \kappa_{3} \kappa_{6}-\kappa_{1}\left(3 \kappa_{3}+4 \kappa_{6}\right)\right)\right)\right)
\end{aligned}
$$

(* The reduced Hamiltonian : *)

$\mathrm{H} 2 \mathrm{r}=$ Simplify[Cancel[H2/. $\left\{\alpha_{11} \rightarrow 0, \alpha_{13} \rightarrow 0, \alpha_{24} \rightarrow 0, \beta_{11} \rightarrow 0, \beta_{21} \rightarrow 0, \beta_{12} \rightarrow 0, \beta_{22} \rightarrow 0, \beta_{13} \rightarrow 0, \beta_{23} \rightarrow 0\right.$,

$\left.\left.\left.\beta_{14} \rightarrow 0, \beta_{24} \rightarrow 0, \beta_{25} \rightarrow 0, \beta_{16} \rightarrow 0\right\}\right]\right] / .\left\{\alpha_{22} \rightarrow 0\right\}$

$\frac{\left(a_{5}^{2}\left(-\kappa_{2}+\kappa_{4}\right)+a_{4}^{2}\left(\kappa_{2}-\kappa_{5}\right)+a_{2}^{2}\left(-\kappa_{4}+\kappa_{5}\right)\right)^{2}}{\left(a_{2}-a_{4}\right)^{2}\left(a_{2}-a_{5}\right)^{2}\left(a_{4}-a_{5}\right)^{2}}+\frac{2\left(a_{5}\left(-\kappa_{2}+\kappa_{4}\right)+a_{4}\left(\kappa_{2}-\kappa_{5}\right)+a_{2}\left(-\kappa_{4}+\kappa_{5}\right)\right)\left(a_{2} a_{5}\left(-a_{2}+a_{5}\right) \kappa_{4}+a_{4}^{2}\left(a_{5} \kappa_{2}-a_{2} \kappa_{5}\right)+a_{4}\left(-a_{5}^{2} \kappa_{2}+a_{2}^{2} \kappa_{5}\right)\right)}{\left(a_{2}-a_{4}\right)^{2}\left(a_{2}-a_{5}\right)^{2}\left(a_{4}-a_{5}\right)^{2}}+$ $\frac{\left(a_{6}^{2}\left(-\kappa_{1}+\kappa_{3}\right)+a_{3}^{2}\left(\kappa_{1}-\kappa_{6}\right)+a_{1}^{2}\left(-\kappa_{3}+\kappa_{6}\right)\right)^{2}}{\left(a_{1}-a_{3}\right)^{2}\left(a_{1}-a_{6}\right)^{2}\left(a_{3}-a_{6}\right)^{2}}+\frac{2\left(a_{6}\left(-\kappa_{1}+\kappa_{3}\right)+a_{3}\left(\kappa_{1}-\kappa_{6}\right)+a_{1}\left(-\kappa_{3}+\kappa_{6}\right)\right)\left(a_{1} a_{6}\left(-a_{1}+a_{6}\right) \kappa_{3}+a_{3}^{2}\left(a_{6} \kappa_{1}-a_{1} \kappa_{6}\right)+a_{3}\left(-a_{6}^{2} \kappa_{1}+a_{1}^{2} \kappa_{6}\right)\right)}{\left(a_{1}-a_{3}\right)^{2}\left(a_{1}-a_{6}\right)^{2}\left(a_{3}-a_{6}\right)^{2}}$

( ${ }^{*}$ For example, check the coincidence Da6 $==$ Da6r where Da6r is obtained from the reduced Hamiltonian $: *$ )

Da6r $=$ Simplify $\left[D\left[\mathrm{H} 2 \mathrm{r}, \kappa_{6}\right]\right]$

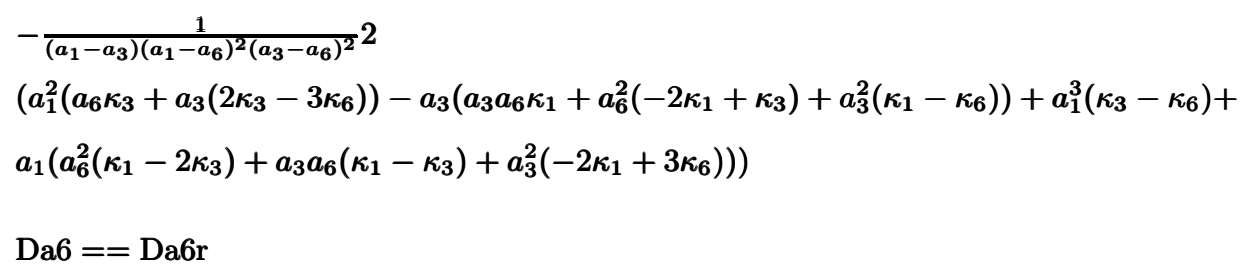

True

(* Next we find out some equations on $\alpha$ under additional requirements on $\kappa$ 's.

We use the notation Aij for $\alpha_{i j} *$ ) 
DA21 $=$ Simplify $\left[\right.$ Series $\left[D\left[\mathrm{H} 2, \beta_{21}\right] / .\left\{\alpha_{11} \rightarrow 0, \alpha_{13} \rightarrow 0, \alpha_{24} \rightarrow 0, \beta_{21} \rightarrow 0, \beta_{12} \rightarrow 0, \beta_{23} \rightarrow 0\right.\right.$, $\left.\left.\left.\beta_{14} \rightarrow 0, \beta_{25} \rightarrow 0, \beta_{16} \rightarrow 0\right\},\left\{\alpha_{22}, 0,0\right\}\right] / \cdot\left\{\alpha_{22} \rightarrow 0, \beta_{22} \rightarrow 0, \beta_{24} \rightarrow 0\right\}\right] / .\left\{\kappa_{3} \rightarrow \kappa_{1}, \kappa_{6} \rightarrow \kappa_{1}\right\}$ $\frac{1}{\left(a_{1}-a_{3}\right)^{3}\left(a_{1}-a_{6}\right)^{3}\left(a_{3}-a_{6}\right)^{2}} 2 \alpha_{21}\left(\left(a_{6}^{2}\left(b_{1}+b_{3}\right)+a_{1}^{2}\left(b_{3}-b_{6}\right)-a_{3}^{2}\left(b_{1}+b_{6}\right)+2 a_{1}\left(-a_{6}\left(b_{1}+b_{3}\right)+\right.\right.\right.$ $\left.\left.a_{3}\left(b_{1}+b_{6}\right)\right)\right)\left(a_{3}^{2}\left(a_{1} \kappa_{1}-a_{6} \kappa_{1}\right)+a_{3}\left(-a_{1}^{2} \kappa_{1}+a_{6}^{2} \kappa_{1}\right)+a_{1}\left(a_{1}-a_{6}\right) a_{6} \kappa_{3}\right)+a_{1}\left(a_{6}^{3}\left(b_{1}+b_{3}\right)-\right.$ $\left.a_{3}^{3}\left(b_{1}+b_{6}\right)+a_{1}^{2}\left(a_{6}\left(b_{1}+b_{3}\right)-a_{3}\left(b_{1}+b_{6}\right)\right)+2 a_{1}\left(-a_{6}^{2}\left(b_{1}+b_{3}\right)+a_{3}^{2}\left(b_{1}+b_{6}\right)\right)\right)\left(a_{6}\left(\kappa_{1}-\kappa_{3}\right)+\right.$ $\left.a_{1}\left(-\kappa_{1}+\kappa_{3}\right)\right)+\left(a_{6}^{3}\left(b_{1}+b_{3}\right)+a_{1}^{3}\left(b_{3}-b_{6}\right)-a_{3}^{3}\left(b_{1}+b_{6}\right)+a_{1}^{2}\left(-a_{6}\left(b_{1}+b_{3}\right)+a_{3}\left(b_{1}+b_{6}\right)\right)+\right.$ $\left.\left.a_{1}\left(-a_{6}^{2}\left(b_{1}+b_{3}\right)+a_{3}^{2}\left(b_{1}+b_{6}\right)\right)\right)\left(a_{6}^{2}\left(\kappa_{1}-\kappa_{3}\right)+a_{1}^{2}\left(-\kappa_{1}+\kappa_{3}\right)\right)\right)$

DA21 $=$ Simplify[DA21/. $\left.\left\{\kappa_{1} \rightarrow K, \kappa_{3} \rightarrow K, \kappa_{6} \rightarrow K\right\}\right]$ (* we will regard to $\mathrm{K}$ as to a constant *) $-\frac{2 K\left(a_{6}^{2}\left(b_{1}+b_{3}\right)+a_{1}^{2}\left(b_{3}-b_{6}\right)-a_{3}^{2}\left(b_{1}+b_{6}\right)+2 a_{1}\left(-a_{6}\left(b_{1}+b_{3}\right)+a_{3}\left(b_{1}+b_{6}\right)\right)\right) \alpha_{21}}{\left(a_{1}-a_{3}\right)^{2}\left(a_{1}-a_{6}\right)^{2}\left(a_{3}-a_{6}\right)}$

(* after a hand made simplification we obtain : *)

$\mathrm{DA} 21:=\frac{2 K\left(\left(a_{1}-a_{3}\right)^{2}\left(b_{1}+b_{6}\right)-\left(a_{1}-a_{6}\right)^{2}\left(b_{1}+b_{3}\right)\right) \alpha_{21}}{\left(a_{1}-a_{3}\right)^{2}\left(a_{1}-a_{6}\right)^{2}\left(a_{3}-a_{6}\right)}$

$\left(*\right.$ FinallyDA21: $\left.=\frac{2 K \alpha_{21}\left(b_{1}+b_{6}\right)}{\left(a_{1}-a_{6}\right)^{2}\left(a_{3}-a_{6}\right)}-\frac{2 K \alpha_{21}\left(b_{1}+b_{3}\right)}{\left(a_{1}-a_{3}\right)^{2}\left(a_{3}-a_{6}\right)} *\right)$

$\mathrm{DA} 23=\operatorname{Simplify}\left[\operatorname{Series}\left[D\left[\mathrm{H} 2, \beta_{23}\right] / .\left\{\alpha_{11} \rightarrow 0, \alpha_{13} \rightarrow 0, \alpha_{24} \rightarrow 0, \beta_{21} \rightarrow 0, \beta_{12} \rightarrow 0, \beta_{23} \rightarrow 0\right.\right.\right.$, $\left.\left.\left.\beta_{14} \rightarrow 0, \beta_{25} \rightarrow 0, \beta_{16} \rightarrow 0\right\},\left\{\alpha_{22}, 0,0\right\}\right] / .\left\{\alpha_{22} \rightarrow 0, \beta_{22} \rightarrow 0, \beta_{24} \rightarrow 0\right\}\right]$

$-\frac{1}{\left(a_{1}-a_{3}\right)^{3}\left(a_{1}-a_{6}\right)^{2}\left(a_{3}-a_{6}\right)^{3}} 2 \alpha_{23}\left(a_{3}\left(a_{6}^{3}\left(b_{1}+b_{3}\right)-a_{1}^{3}\left(b_{3}+b_{6}\right)+a_{3}^{2}\left(a_{6}\left(b_{1}+b_{3}\right)-a_{1}\left(b_{3}+b_{6}\right)\right)-\right.\right.$ $\left.2 a_{3}\left(a_{6}^{2}\left(b_{1}+b_{3}\right)-a_{1}^{2}\left(b_{3}+b_{6}\right)\right)\right)\left(a_{6}\left(-\kappa_{1}+\kappa_{3}\right)+a_{3}\left(\kappa_{1}-\kappa_{6}\right)+a_{1}\left(-\kappa_{3}+\kappa_{6}\right)\right)+\left(a_{6}^{3}\left(b_{1}+\right.\right.$ $\left.b_{3}\right)+a_{3}^{3}\left(b_{1}-b_{6}\right)-a_{1}^{3}\left(b_{3}+b_{6}\right)+a_{3}^{2}\left(-a_{6}\left(b_{1}+b_{3}\right)+a_{1}\left(b_{3}+b_{6}\right)\right)+a_{3}\left(-a_{6}^{2}\left(b_{1}+b_{3}\right)+a_{1}^{2}\left(b_{3}+\right.\right.$ $\left.\left.\left.b_{6}\right)\right)\right)\left(a_{6}^{2}\left(-\kappa_{1}+\kappa_{3}\right)+a_{3}^{2}\left(\kappa_{1}-\kappa_{6}\right)+a_{1}^{2}\left(-\kappa_{3}+\kappa_{6}\right)\right)+\left(a_{6}^{2}\left(b_{1}+b_{3}\right)+a_{3}^{2}\left(b_{1}-b_{6}\right)-a_{1}^{2}\left(b_{3}+b_{6}\right)-\right.$ $\left.\left.2 a_{3}\left(a_{6}\left(b_{1}+b_{3}\right)-a_{1}\left(b_{3}+b_{6}\right)\right)\right)\left(a_{1} a_{6}\left(-a_{1}+a_{6}\right) \kappa_{3}+a_{3}^{2}\left(a_{6} \kappa_{1}-a_{1} \kappa_{6}\right)+a_{3}\left(-a_{6}^{2} \kappa_{1}+a_{1}^{2} \kappa_{6}\right)\right)\right)$

$\left(*\right.$ Finally DA23 $\left.=\frac{\left.2 \mathrm{~K} \alpha_{23}\left(\left(a_{1}-a_{3}\right)^{2}\left(b_{3}+b_{6}\right)-\left(a_{3}-a_{6}\right)^{2}\left(b_{1}+b_{3}\right)\right)\right)}{\left(a_{1}-a_{3}\right)^{2}\left(a_{1}-a_{6}\right)\left(a_{3}-a_{6}\right)^{2}}=\frac{2 \mathrm{~K} \alpha_{23}\left(b_{3}+b_{6}\right)}{\left(a_{1}-a_{6}\right)\left(a_{3}-a_{6}\right)^{2}}-\frac{2 \mathrm{~K} \alpha_{23}\left(b_{1}+b_{3}\right)}{\left(a_{1}-a_{3}\right)^{2}\left(a_{1}-a_{6}\right)} *\right)$

\section{REFERENCES}

[1] Gawędzki, K., Tran-Ngog-Bich, P. Hitchin systems at low genera. arXiv:hep-th/9803101

[2] Van Geemen, B., Previato, E. On the Hitchin system. Duke Math. J., V. 85., p.659-683 (1996).

[3] Krichever, I.M. Vector bundles and Lax equations on algebraic curves. Comm. Math. Phys. 229, 229-269 (2002). hep-th/0108110

[4] Prasolov, V. Polynomials. Moscow, MCNMO, 2001, 335 p. (in Russian).

[5] Sheinman, O.K. Current algebras on Riemann surfaces, De Gruyter Expositions in Mathematics, 58, Walter de Gruyter GmbH \& Co, Berlin-Boston, 2012, ISBN: 978-3-11-026452-4, $150 \mathrm{pp}$.

[6] Sheinman, O.K., Lax operators algebras and gradings on semisimple Lie algebras, Dokl. Math., 91:2 (2015), 160162 
[7] Sheinman, O.K. Hierarchies of finite-dimensional Lax equations with a spectral parameter on a Riemann surface, and semi-simple Lie algebras. Theoret. and Math. Phys., 185:3 (2015), $1816-1831$.

[8] Sheinman, O.K. Semi-simple Lie algebras and Hamiltonian theory of finite-dimensional Lax equations with the spectral parameter on a Riemann surface. Proc. Steklov Inst. Math., 290 (2015), 178-188.

[9] Sheinman, O.K. Global current algebras and localization on Riemann surfaces, Moscow Math. Journ., V. 15, n. 4, (2015), p. 833-846.

[10] Sheinman, O.K. Lax operator algebras and integrable systems. Russian Math. Surveys, 71:1 (2016), 109-156. arXiv: 1602.04320

[11] Tyurin, A.N. Classification of vector bundles over an arbitrary genus algebraic curve. Amer. Math. Soc. Transl. Ser. 2, 63 (1967),

Department of Geometry and Topology, Steklov Mathematical Institute, Moscow, AND INDEPENDENT UNIVERSity OF Moscow 